\title{
Belostomatidae (Heteroptera: Nepomorpha) held in the Invertebrate Collection of the Instituto Nacional de Pesquisas da Amazônia, Manaus, Brazil: inventory and new distributional records
}

\author{
Thaís Melo de Almeida ${ }^{1,3}$; Fabiano Stefanello ${ }^{2}$ \& Neusa Hamada, \\ ${ }^{1}$ Instituto Nacional de Pesquisas da Amazônia (INPA), Coordenação de Biodiversidade (COBIO), Programa de Pós-Graduação em Entomologia \\ (PPG-ENT). Manaus, AM, Brasil. \\ 2 Universidade de São Paulo (USP), Faculdade de Filosofia, Ciências e Letras de Ribeirão Preto (FFCLRP), Departamento de Biologia, Laboratório \\ de Biologia Comparada e Abelhas (LBCA). Ribeirão Preto, SP, Brasil. ORCID: http://orcid.org/0000-0002-9622-8311. E-mail: stefanellof@usp.br \\ 3 ORCID: http://orcid.org/0000-0002-0282-1043. E-mail: thais.mel020@gmail.com (corresponding author) \\ ${ }^{4}$ ORCID: http://orcid.org/0000-0002-3526-5426. E-mail: nhamada@inpa.gov.br
}

\begin{abstract}
Belostomatidae is a family of giant water bugs with 52 valid species in Brazil. Here, we list 33 valid species housed in the Invertebrates Collection of the Instituto Nacional de Pesquisas da Amazônia - INPA, Manaus, Brazil. Of the total species examined, 26 had their distributional ranges increased (new records provided in parentheses): Belostoma amazonum Estévez \& Polhemus (Mato Grosso and Roraima); B. aurivillianum (Montandon) (Rondônia); B. bosqi De Carlo (Maranhão); B. candidulum Montandon (Mato Grosso do Sul); B. carajaensis Ribeiro \& Estévez (Amazonas); B. dallasi De Carlo (Bahia and Pará); B. dentatum (Mayr) (Roraima); B. denticolle Montandon (Roraima); B. dilatatum (Dufour) (Maranhão); B. discretum Montandon (Rio Grande do Sul); B. elongatum Montandon (Paraná); B. estevezae Ribeiro \& Alecrim (Amazonas); B. fittkaui De Carlo (Roraima); B. gestroi Montandon (Pará); B. guianae Lauck (Brazil, in Roraima); B. micantulum (Stål) (Maranhão, Rio Grande do Norte, Rondônia and Roraima); B. minusculum (Uhler) (Brazil, in Amazonas, Pará and Rio Grande do Norte); B. parvum Estévez \& Polhemus (Amazonas and Roraima); B. plebejum (Stål) (Roraima); B. pygmeum (Dufour) (Pará); B. ribeiroi De Carlo (Mato Grosso do Sul); B. stollii (Amyot \& Serville) (Roraima); Horvathinia pelocoroides Montandon (Rondônia); Lethocerus annulipes (HerrichSchaeffer) (Maranhão, Paraíba and Paraná); L. delpontei De Carlo (Amazonas and Pará) and L. maximus De Carlo (Acre, Amapá, Amazonas and Roraima). In addition, we provide a list of type and non-type specimens of Belostomatidae from INPA's collection identified to the species level, photographs of these species and relevant taxonomic notes, when available.
\end{abstract}

Key-Words. Aquatic insect; Giant water bugs; INPA; Taxonomy.

\section{INTRODUCTION}

Belostomatidae Leach, 1815 comprises 11 genera and approximately 150 species, most of which are reported from the New World (Polhemus, 1995; Schuh \& Slater, 1995; Perez-Goodwyn, 2006; Estévez \& Ribeiro, 2011; Moreira et al., 2011; Ribeiro et al., 2017; Ribeiro et al., 2018). The life cycle of species in this family are fully dependent on freshwater habitats, mainly permanent or temporary ponds, streams and the edges of rivers. In Brazil, 52 species are currently recorded, distributed among four genera: Belostoma Latreille, 1807, Horvathinia Montandon, 1911, Lethocerus Mayr, 1853 and Weberiella De Carlo, 1966 (Estévez \& Polhemus, 2001; Ribeiro, 2007; Ribeiro \& Alecrim, 2008; Ribeiro \& Estévez, 2009; Moreira et al., 2011; Ribeiro et al., 2018).
Currently, Belostomatidae has been recorded in all Brazilian states except Pernambuco and Sergipe, but the lack of reports for these two states undoubtedly is the result of a lack of sampling (Ribeiro et al., 2018). The Brazilian region with the largest number of species recorded in the family is the South (with 32 species) and the region with the smallest number is the Northeast (with 14 species) (Ribeiro et al., 2018). Biological collections in several Brazilian institutions have many unidentified specimens (Magalhães et al., 2005) or, with specimens identified only to the genus or family level, including Belostomatidae specimens. This fact is common to several other taxa and, according to Taddei et al. (1999), biases our understanding of diversity in the region, especially because some of these specimens can represent new species. There are several reasons for 
A

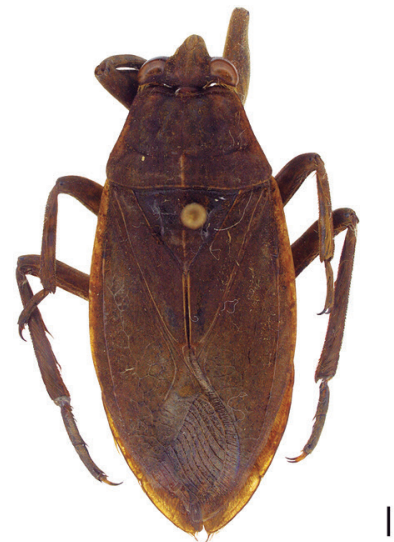

D

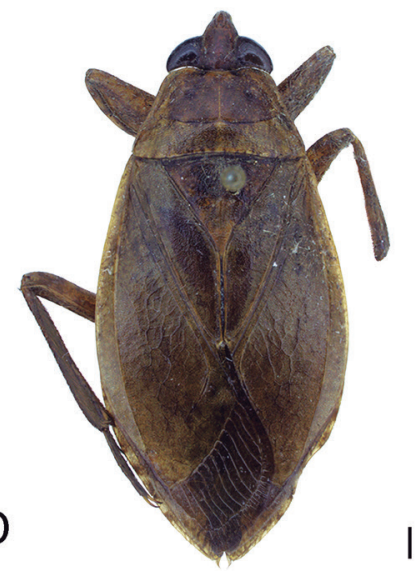

G

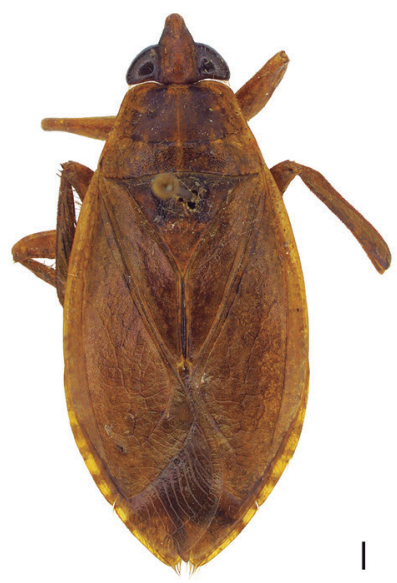

$\mathrm{H}$

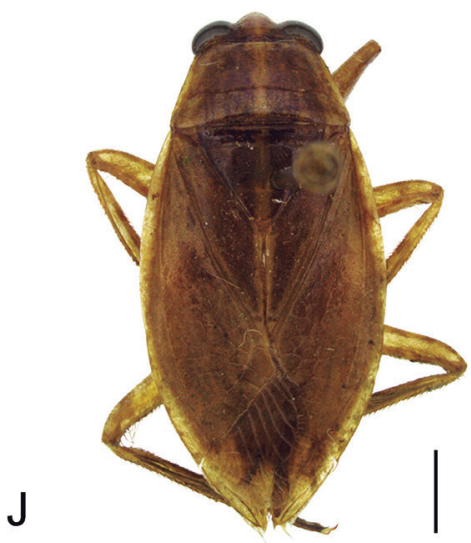

B

E

K

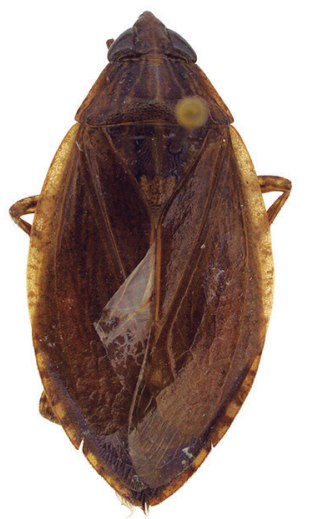

C
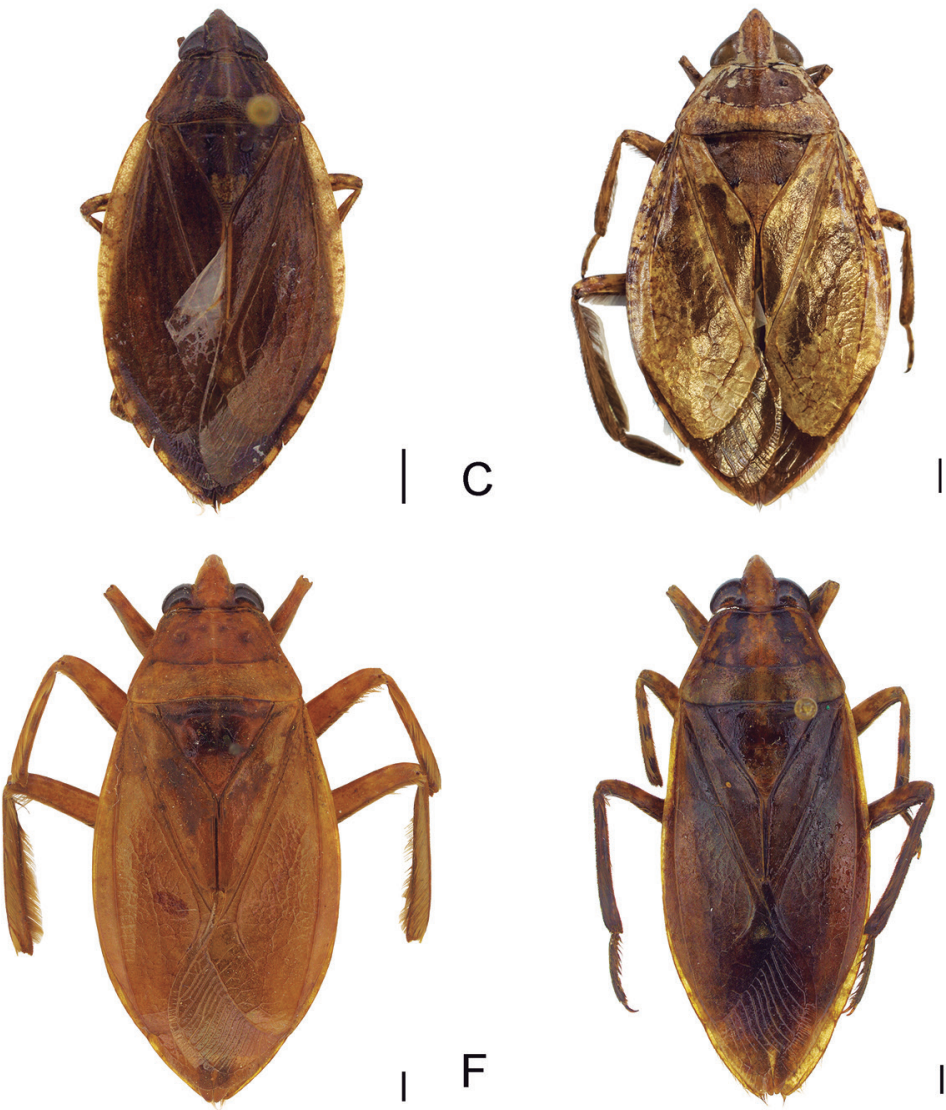

F
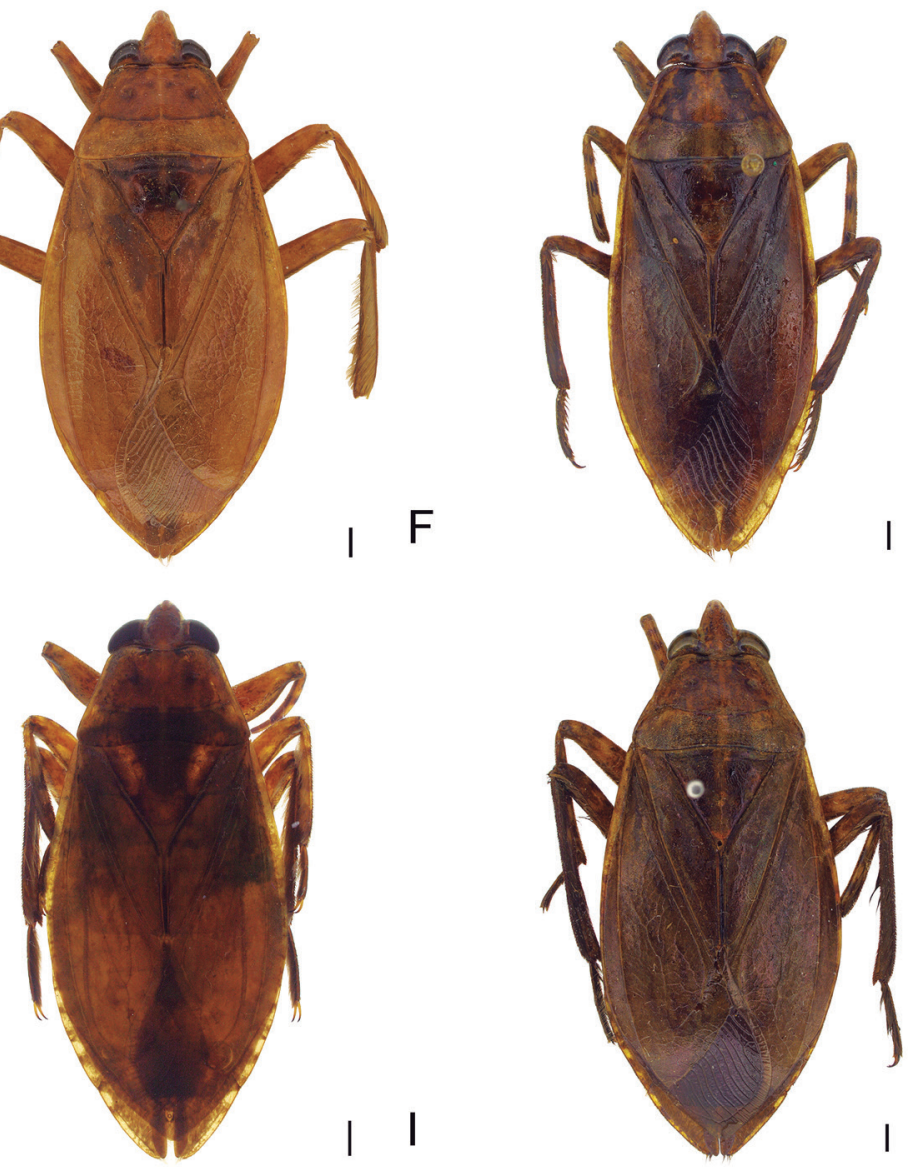

I
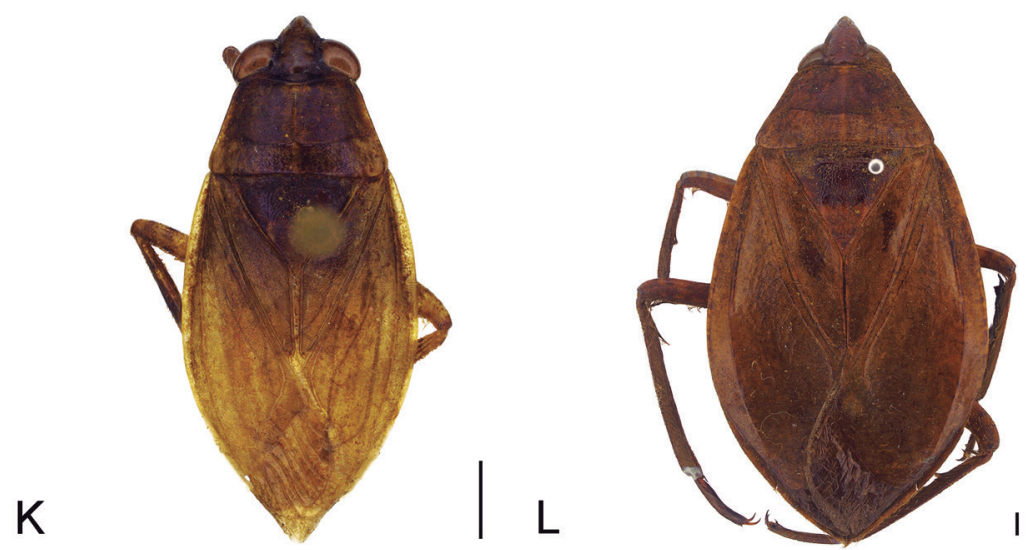

Figure 1. Species of Belostomatidae. Dorsal view. $(A)=$ Belostoma aurivilianum (Male, from Amazonas). $(B)=B$. bosqi (Female, from Ceará). $(C)=B$. costalimai (Male, from Pará). (D) = B. dallasi (Male, from Bahia). $(\mathrm{E})=$ B. dentatum (Male, from Amazonas). $(\mathrm{F})=$ B. elongatum (Male, from Mato Grosso). $(\mathrm{G})=$ B. foveolatum (Male, from Ceará). $(\mathrm{H})=$ B. guianae (Female, from Roraima). $(\mathrm{I})=$ B. harrisi (Male, Amazonas). $(\mathrm{J})=$ B. amazonum (Male, Amazonas). $(\mathrm{K})=B$. denticolle (Male, Amazonas). $(\mathrm{L})=$ B. dilatatum (Male, Maranhão). Scale bar: $2 \mathrm{~mm}$. 

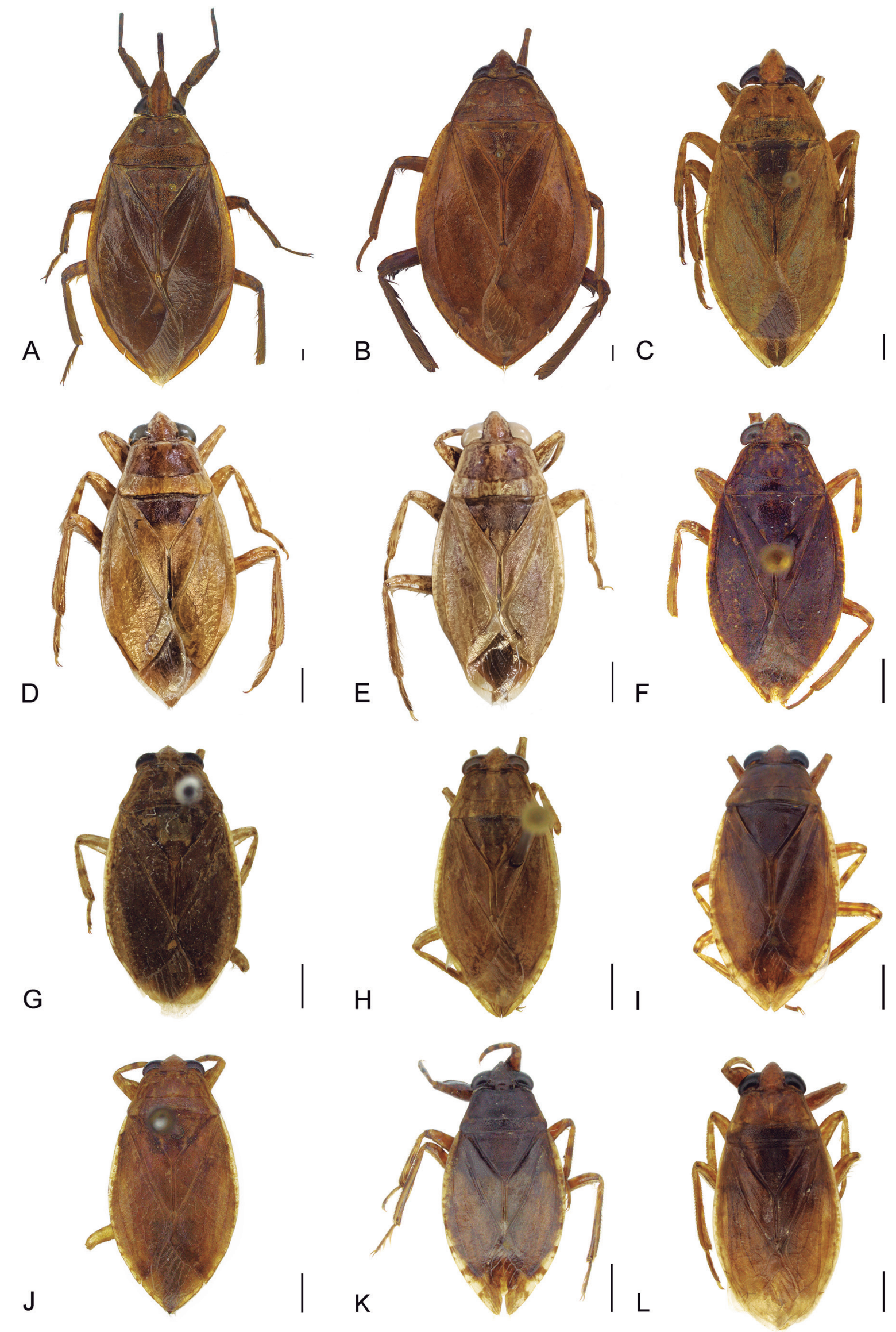

Figure 2. Species of Belostomatidae. Dorsal view. $(A)=$ Belostoma ripicolum - paratype (Male, Rio Grande do Sul). $(B)=B$. gestroi (Male, Amazonas). (C) = B. discretum (Male, Amazonas). $(\mathrm{D})=$ = B. candidulum (Male, from Mato Grosso do Sul). $(\mathrm{E})=$. carajaensis (Male, Amazonas). $(\mathrm{F})=$ B. estevezae (Female, Amazonas). (G) = B. micantulum (Male, Amazonas). (H) = B. minusculum (Male, Amazonas). $(\mathrm{I})=$ B. nessimiani - holotype (Male, Amazonas). (J) = B. nicaem (Female, Amazonas). $(\mathrm{K})=$ B. parvum (Female, Amazonas). $(\mathrm{L})=$ B. plebejum (Male, Amazonas). Scale bar: $2 \mathrm{~mm}$. 


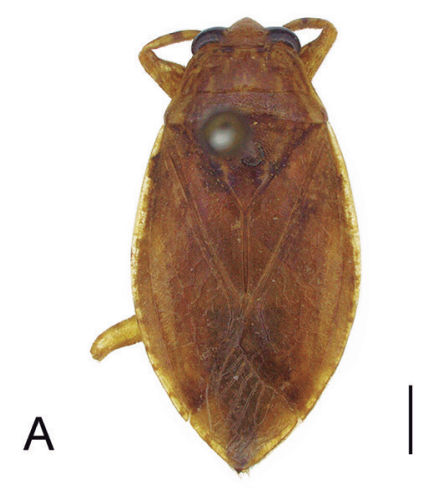

B
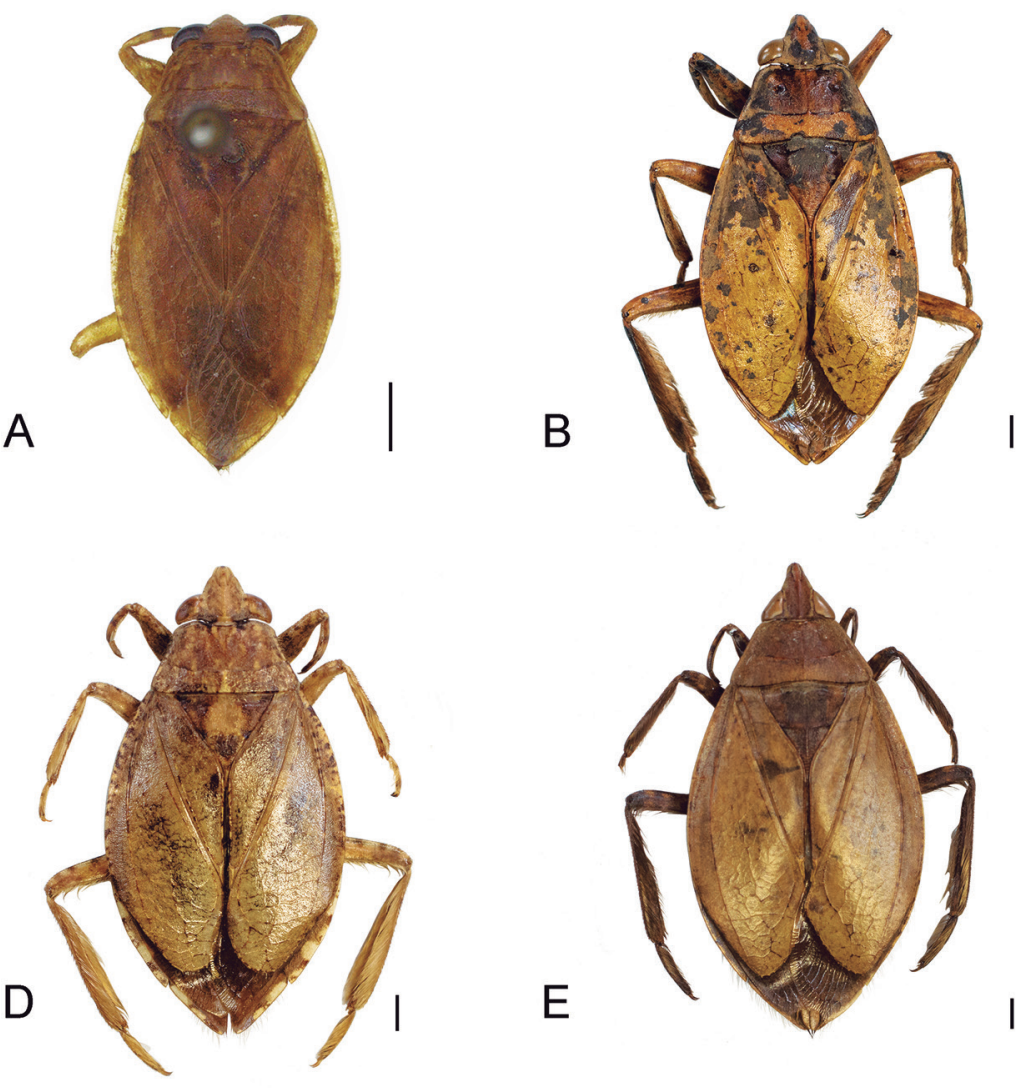

E

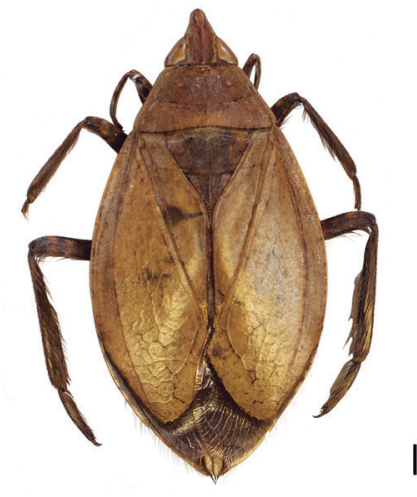

I F
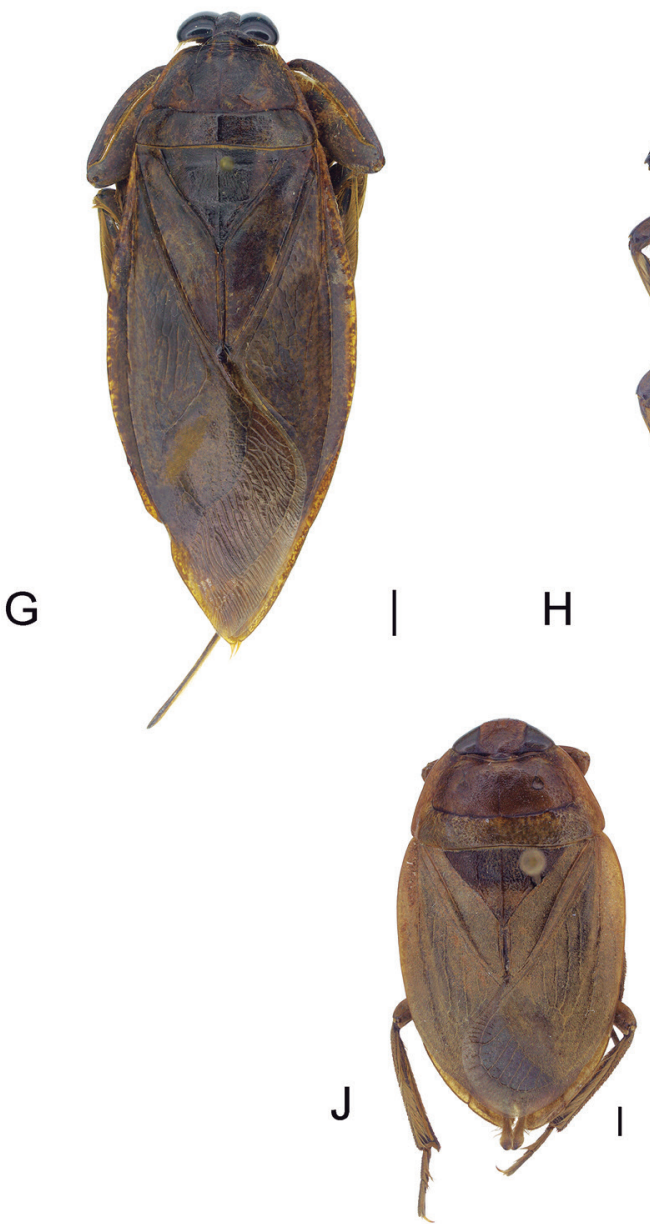

$\mathrm{H}$

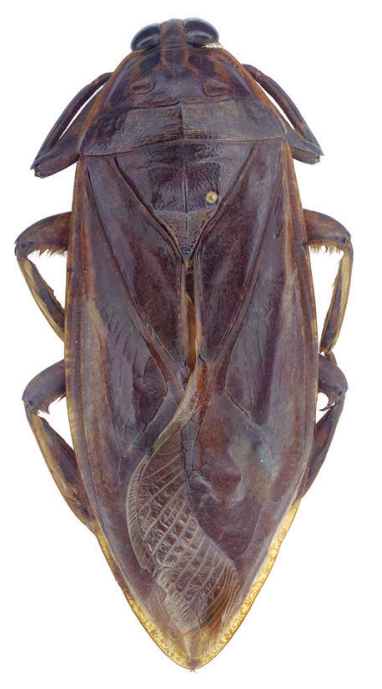

I I
C
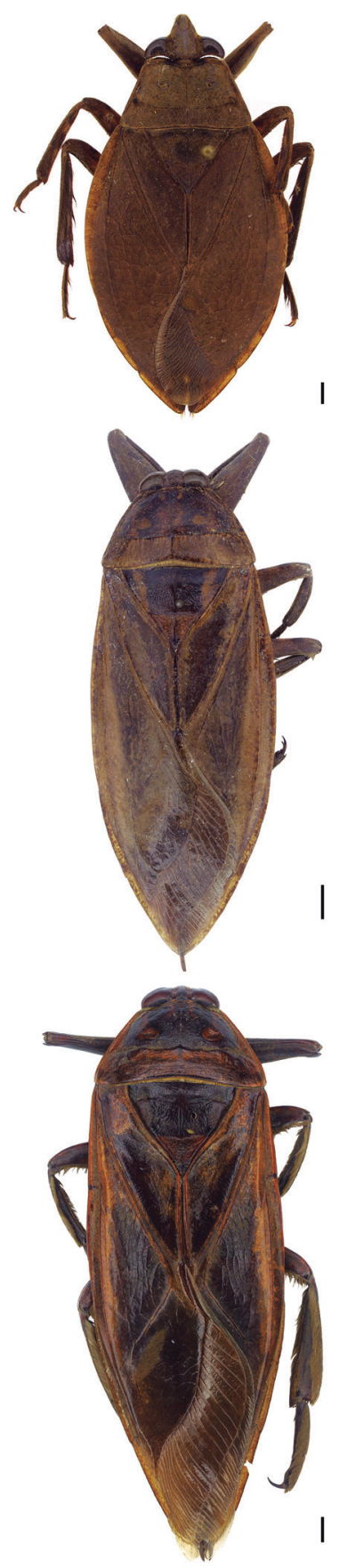

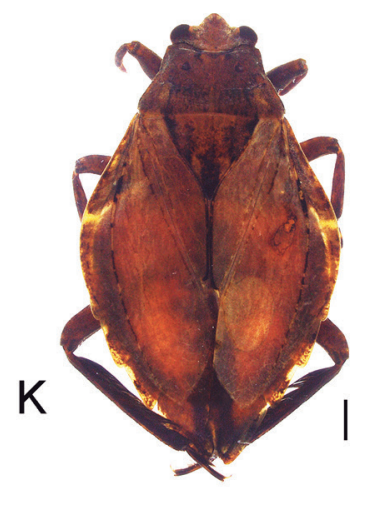

Figure 3. Species of Belostomatidae. Dorsal view. $(\mathrm{A})=$ Belostoma pygmeum (Male, Amazonas). (B) = B. ribeiroi (Female, from Mato Grosso do Sul). (C) = B. stollii (Male, Amazonas). ( $(\mathrm{D})=$ B. bachmanni (Female, from Amazonas). $(\mathrm{E})=$ B. fittkaui (Male, from Amazonas). $(\mathrm{F})=$ Lethocerus annulipes (Male, from Amazonas). $(\mathrm{G})=$ L. delpontei (Male, from Amazonas). $(\mathrm{H})=$ L. maximus (Male, from Roraima). $(\mathrm{I})=$ L. paraensis - holotype (Female, from Pará). $(\mathrm{J})=$ Horvathinia pelocoroides (Male, from Rondônia). (K) = Weberiella rhomboides (Male, from Rondônia). Scale bar: (A-E, J-K): 2 mm, (F-I): 5 mm. 
the lack of species-level identification of the specimens housed in taxonomic collections, the main one being the lack of trained taxonomists.

The Invertebrate Collection of the Instituto Nacional de Pesquisas da Amazônia (INPA), located in Manaus, Amazonas state houses three type specimens of Belostomatidae: Belostoma nessimiani Ribeiro \& Alecrim, 2008, Belostoma ripicolum Lanzer-de-Souza, 1992 junior synonym of Belostoma dilatatum (Dufour, 1863), according to Ribeiro (2007), and Lethocerus paraensis Lanzerde-Souza, 1991 junior synonym of Lethocerus maximus De Carlo, 1938, according to Perez-Goodwyn (1997). In addition to these type specimens, INPA's collection houses many non-type specimens that are unidentified, mainly representing the North region, with some species being exclusive to the Amazon region.

The objectives of this study are to identify at the species level all the giant water bugs housed in the Invertebrate Collection of INPA and provide a list with type and non-type specimens in this collection, with relevant taxonomic notes (when available), photographs of all species listed, and new distributional records.

\section{MATERIAL AND METHODS}

We examined non-type and type specimens, both pinned and in $70 \%$ ethyl alcohol, that are housed in the Invertebrate Collection of INPA. The following papers were used for the identification of these specimens to the species level: De Carlo (1958), Schnack (1976), Estévez \& Polhemus (2001), Ribeiro (2005), Schnack \& Estévez (2005), Perez-Goodwyn (2006), Estévez \& Polhemus (2007), Ribeiro (2007), Ribeiro \& Alecrim (2008), Ribeiro \& Estévez (2009), Estévez \& Ribeiro (2011) and Ribeiro et al. (2017).

One representative of each species was photographed using a stereoscopic microscope (Leica M165C), with a digital camera DFC420 and Leica Application Suite V3.6 digital image processing software. Comments were added indicating the new records and relevant taxonomic notes.

The maps were made in the DIVA-GIS software, version 7.5 .

\section{RESULTS AND DISCUSSION}

A total of 628 Belostomatidae specimens were examined belonging to 33 species, 29 of which belonged to the genus Belostoma (distributed in 11 species groups), three species belonged to the genus Lethocerus, and two genera had only one species each: Horvathinia pelocoroides Montandon, 1911 and Weberiella rhomboides (Menke, 1965). The species are listed below, including type specimens deposited in the collection. The most abundant species was $B$. discretum Montandon, 1903b - with 257 specimens and the least abundant, with one specimen each, were $B$. carajaensis Ribeiro \& Estévez, 2009, B. costalimai De Carlo, 1938, B. estevezae Ribeiro \& Alecrim, 2008, B. nicaem Estevez \& J. Polhemus, 2007 and $W$. rhomboides. New geographic distributional re- cords are provided for 26 species, including the following states: Acre (AC), Amapá (AP), Amazonas (AM), Bahia $(B A)$, Ceará (CE), Maranhão (MA), Mato Grosso (MT), Mato Grosso do Sul (MS), Pará (PA), Paraíba (PB), Paraná (PR), Rio Grande do Norte (RN), Rio Grande do Sul (RS), Roraima (RR) and Rondônia (RO). The state with more new records was Roraima with nine species. In addition, we report two new records for Brazil - Belostoma guianae Lauck, 1962 and B. minusculum (Uhler, 1884), increasing the number of giant water bugs in the country to 54 .

Knowledge of the diversity of Belostomatidae species from the Invertebrate Collection of INPA is summarized in this study. The taxonomic information and new geographic records mentioned here could provide input to biodiversity and biogeographical studies, as well as environmental monitoring.

\section{List of species of Belostomatidae in the Invertebrate Collection of INPA \\ Belostoma Latreille, 1807 \\ Aurivillianum group sensu Lauck (1963)}

Diagnose: Pronotum and scutellum elevated with a distinct median carina; pilosity covering about threefourths the connexivum.

\section{Belostoma aurivillianum (Montandon, 1899) (Figs. 1A, 4A)}

Known distribution: BOLIVIA: Santa Cruz (Nieser, 1975), unkown Department (Lauck, 1963). BRAZIL: Goiás (Menke \& Lauck, 1962), Mato Grosso (Ribeiro, 2005), Minas Gerais (Nieser \& Melo, 1997), Pará (Lauck, 1963), Paraná (Ribeiro, 2005), Rio de Janeiro (Ribeiro, 2005), Rio Grande do Sul (Lanzer, 1976), Roraima (Nieser, 1975), São Paulo (Lauck, 1963), unkown State (Montandon, 1899). COLOMBIA: unknown Department (Montandon, 1899). PARAGUAY: Cororó (De Carlo, 1966). SURINAM: Nickerie (Nieser, 1975). VENEZUELA: unkown State (Montandon, 1899).

Material examined: 1 male and 1 female, pinned, BRAZIL, Mato Grosso, Vila Bela da Santíssima Trindade municipality, Matrinchã stream, mobile light trap, 12.VII.2010, J.A. Rafael, T.K. Krolow, R. Machado colls., T.M. Almeida, 2018 det. 1 male, pinned, Rondônia, near Pimenta Bueno municipality, Guaporé river, $12^{\circ} 13^{\prime} 19^{\prime \prime} \mathrm{S}$ 60³2'44"W, light trap, 23.IV.2006, J.A. Rafael \& F.F. Xavier Fo colls., T.M. Almeida, 2017 det. 1 female, pinned, Roraima, Amajari municipality, Serra do Tepequém, $03^{\circ} 46^{\prime} 31^{\prime \prime} \mathrm{N}$ $61^{\circ} 04^{\prime} 05^{\prime \prime} \mathrm{W}$, mixed light and BLB, 01.IX.2004, J.A. Rafael, F.F. Xavier Fo, A. Silva \& F. Leo colls., T.M. Almeida, 2017 det.; 1 female, pinned, Boa Vista municipality, 06.VII.1977, N.D. Penny coll., 0020938, T.M. Almeida, 2017 det.; 1 male, pinned, Caracaraí municipality, 13.V.1977, N.D. Penny coll., 0020902, T.M. Almeida, 2017 det.; 1 female, pinned, Pacaraima municipality, $04^{\circ} 27^{\prime} 04^{\prime \prime} \mathrm{N} 61^{\circ} 07^{\prime} 56^{\prime \prime} \mathrm{W}$, mixed light trap, 07.VII.1996, B.R. Teles \& F.F. Xavier Fo colls., 


\section{Belostoma bosqi De Carlo, 1932}

(Figs. 1B, 4B)

ethyl alcohol 70\%, Amajari municipality, Uraricoera river, Maracá Ecological Station, light trap, 22.IX.1987, team Nhamenhame colls., M.E. Lanzer-de-Souza, 1987 det.

Notes: This is the first record of $B$. aurivillianum to Rondônia state (Brazil).

\section{Bergi group sensu Lauck (1962)}

Diagnose: Rostrum with segment I distinctly longer than segment II; eye acutely triangular, outer margin nearly straight.
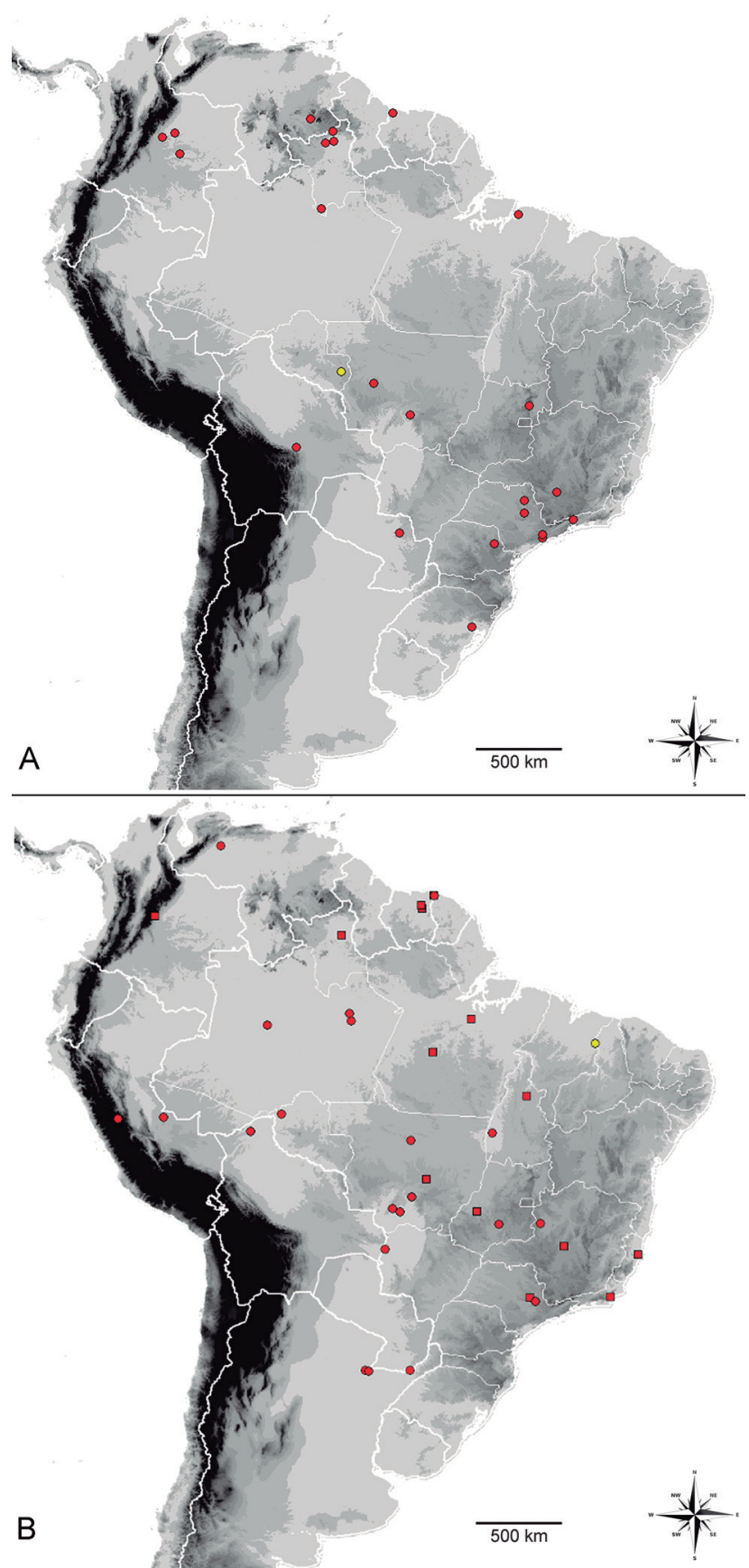

Figure 4. Distribution of Belostomatidae in South America. Red symbol known records, yellow symbol - new records. $(A)=$ Belostoma aurivillianum (circle). (B) = B. bosqi (circle), B. costalimai (square).
Known distribution: ARGENTINA: Chaco (De Carlo, 1930), Corrientes (Lauck, 1964), Missiones (De Carlo, 1932). BOLIVIA: Pando (Lauck, 1964), Santa Cruz (Lauck, 1964). BRAZIL: Amazonas (Nieser, 1975), Goiás (Menke \& Lauck, 1962), Mato Grosso (Lauck, 1964), Mato Grosso do Sul (De Carlo, 1930), Minas Gerais (Ribeiro et al., 2018), Rondônia (Ribeiro et al., 2018), São Paulo (Ribeiro et al., 2018), Tocantins (Lauck, 1964). PERU: Huánuco (Lauck, 1964), Ucayali (Lauck, 1964). SURINAM: Marowijne (Nieser, 1975). VENEZUELA: Portuguesa (Lauck, 1964).

Material examined: 1 female, pinned, BRAZIL, Amazonas, Manaus municipality, BR 174, km 45, Cabeça Branca stream, mixed light trap, 08.VIII.2001, C.A.S. Azevedo coll., Hemiptera - Belostomatidae, C.A.S. Azevedo, 27.VIII.2001 det.; 2 males, pinned, $90 \mathrm{~km}$ from Manaus municipality, ZF-2 Reserve, $\mathrm{km} \mathrm{14}$, tower, $02^{\circ} 35^{\prime} 21^{\prime \prime} \mathrm{S} 60^{\circ} 06^{\prime} 55^{\prime \prime} \mathrm{W}$, mixed light and BLB, 13-16.VIII.2004, J.A. Rafael, F.F. Xavier Fo, A.R. Ururahy, A. Silva F \& S. Trovisco colls., T.M. Almeida, 2013 det. 1 male, pinned,

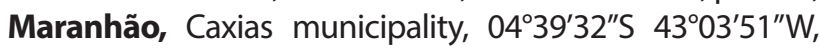
100 m, 19.V.2007, J.A. Rafael, F.F. Xavier Fo \& F.L. Oliveira colls., T.M. Almeida, 2013 det. 5 males and 1 female, pinned, Mato Grosso, Cáceres municipality, 16 $09^{\prime} 30^{\prime \prime} \mathrm{S} 57^{\circ} 07^{\prime} 05^{\prime \prime} \mathrm{W}$, light trap, 30.IV.2006, J.A. Rafael \& F.F. Xavier Fo colls., T.M. Almeida, 2013 det.; 1 female, pinned, Chapada dos Guimarães municipality, Salgadeira, $15^{\circ} 21^{\prime} 23^{\prime \prime} \mathrm{S} 55^{\circ} 49^{\prime} 48^{\prime \prime} \mathrm{W}$, light trap, 05.V.2006, J.A. Rafael \& F.F. Xavier Fo colls., T.M. Almeida, 2013 det.; 2 males and 1 female, pinned, Poconé municipality, Ipiranga farm, $16^{\circ} 23^{\prime} 06^{\prime \prime} \mathrm{S} 56^{\circ} 35^{\prime} 28^{\prime \prime} \mathrm{W}$, light trap, 02.V.2006, J.A. Rafael \& F.F. Xavier Fo colls., T.M. Almeida, 2013 det.

Notes: This is the first record of $B$. bosqi to Maranhão state (Brazil).

\section{Belostoma costalimai De Carlo, 1938 (Figs. 1C, 4B)}

Known distribution: BRAZIL: Espírito Santo (Ribeiro, 2005), Goiás (Menke \& Lauck, 1962), Mato Grosso (Lauck, 1964), Minas Gerais (Nieser \& Melo, 1997), Pará (Ribeiro, 2000), Rio de Janeiro (De Carlo, 1938), Roraima (Ribeiro, 2000), São Paulo (De Carlo, 1938), Tocantins (Lauck, 1964). COLOMBIA: Villavicencio (Roback \& Nieser, 1974). SURINAM: Brokopondo (Nieser, 1975), Kabelstation (De Carlo, 1960), Marowijne (Nieser, 1975).

Material examined: 1 male, ethyl alcohol 70\%, BRAZIL, Pará, Altamira municipality, Poção stream, near Xingu river, 11.X.1992, F. Stefanello, 2018 det.

\section{Dentatum group sensu Nieser (1975)}

Diagnose: Beak with segment I usually longer than Il; ventral diverticulum of genitalia with a large prominent conical protuberance and an apical prominance in lateral view. 


\section{Belostoma dallasi De Carlo, 1930 (Figs. 1D, 5A)}

Known distribution: ARGENTINA: Entre Ríos (Ribeiro et al., 2017), Formosa (Ribeiro et al., 2017), Jujuy (Ribeiro et al., 2017), Salta (De Carlo, 1930), Tucumán (De Carlo, 1930). BRAZIL: Ceará (Ribeiro, 2007), Goiás (Menke \& Lauck, 1962), Minas Gerais (Nieser \& Melo, 1997), Rio de Janeiro (Ribeiro et al., 2017), unknow State (De Carlo, 1960). COLOMBIA: unknow Department (Ribeiro et al., 2017).

Material examined: 1 male and 3 females, pinned, BRAZIL, Bahia, Encruzilhada municipality, $800 \mathrm{~m}$,
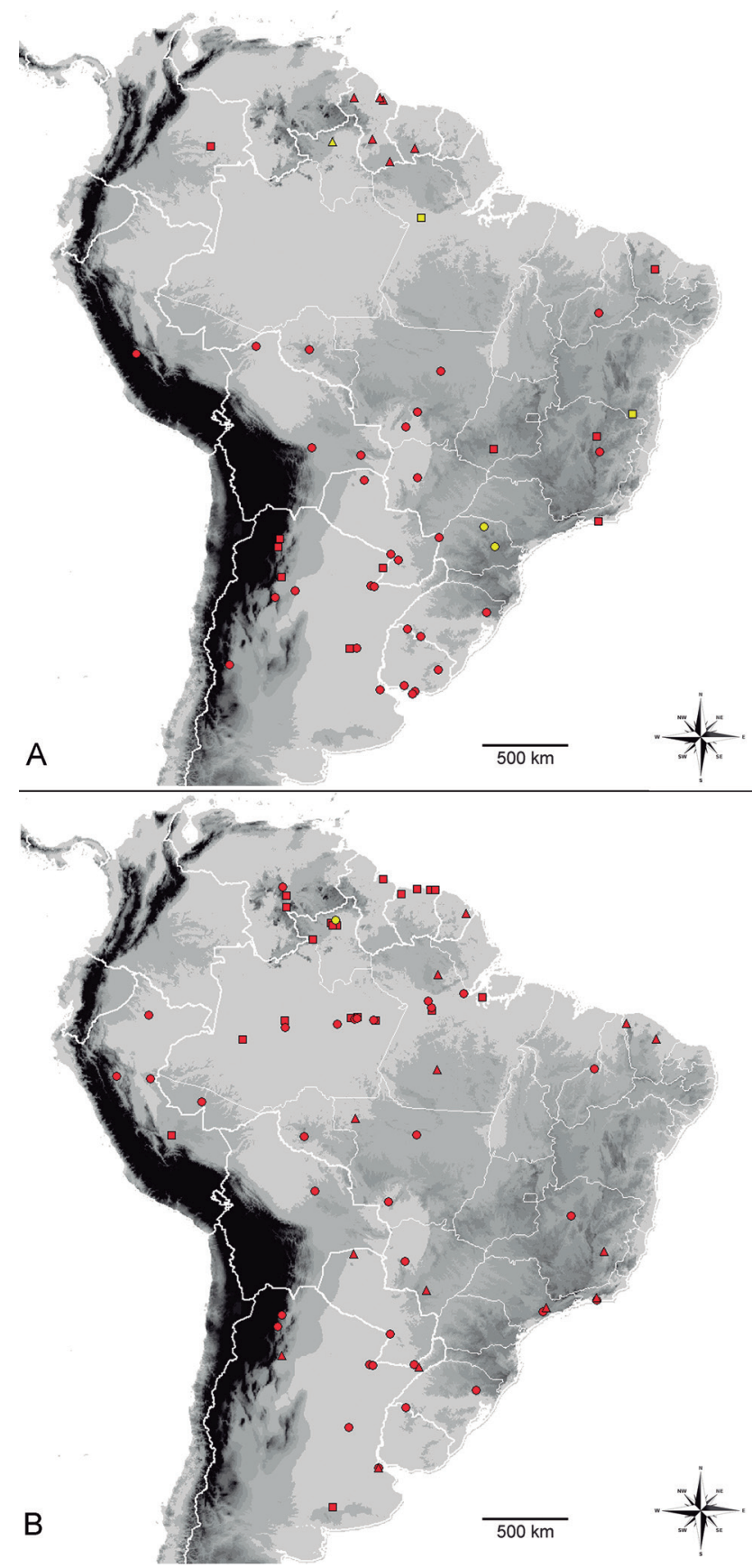

Figure 5. Distribution of Belostomatidae in South America. Red symbol - known records, yellow symbol - new records. $(A)=$ Belostoma dallasi (square), B. elongatum (circle), B. guianae (triangle). (B) = B. dentatum (circle), B. foveolatum (triangle), B. harrisi (square). $15^{\circ} 32^{\prime} 25^{\prime \prime S} 40^{\circ} 50^{\prime} 12^{\prime \prime}$ W, light trap, 10-12.XII.2007, J.A. Rafael, P.C. Grossi \& D.R. Parizotto colls., T.M. Almeida, 2013 det. 1 female, pinned, Pará, Óbidos municipality, 0020871, T.M. Almeida, 2013 det.

Notes: This is the first record of $B$. dallasi to Bahia and Pará states (Brazil).

\section{Belostoma dentatum (Mayr, 1863)}

(Figs. 1E, 5B)

Known distribution: ARGENTINA: Buenos Aires (De Carlo, 1930), Chaco (De Carlo, 1930), Corrientes (De Carlo, 1930), Entre Ríos (De Carlo, 1930), Jujuy (Morrone et al., 2004), Misiones (De Carlo, 1930), Salta (De Carlo, 1938), Santa Fé (De Carlo, 1938). BOLIVIA: unknow Departament (De Carlo, 1930). BRAZIL: Acre (Ribeiro, 2005), Amazonas (Nieser, 1975), Mato Grosso (Ribeiro, 2005), Mato Grosso do Sul (Nieser, 1975), Minas Gerais (De Carlo, 1938), Pará (Nieser, 1975), Piauí (Ribeiro, 2005), Rio de Janeiro (Nieser, 1975), Rio Grande do Sul (Lauck, 1962), Rondônia (Nieser, 1975), São Paulo (Ribeiro, 2005), unknow State (Mayr, 1863). PARAGUAY: Asunción (De Carlo, 1938), Central (Montandon, 1895), Concepción (Chernoff et al., 2001), Guairá (Nieser, 1975). PERU: Arequipa (Lauck, 1962), Junin (Ribeiro et al., 2017), Loreto (Lauck, 1962), San Martin (Lauck, 1962). URUGUAY: Artigas (Ruffinelli \& Pirán, 1959). VENEZUELA: unknow State (De Carlo, 1930).

Material examined: 1 female, ethyl alcohol 70\%, BRAZIL, Amazonas, Grande lake, near Purus river, 1854, 30.XI.1986, F.A. Peralta coll., T.M. Almeida, 2014 det.; 1 female, pinned, Iranduba municipality, Marchantaria island, 26.III.1976, E. Castellon coll., T.M. Almeida, 2013 det.; 1 female, pinned, Iranduba municipality, VIII.1956, 0020907, T.M. Almeida, 2013 det.; 1 female and 2 males, pinned, Iranduba municipality, $03^{\circ} 17^{\prime} 38^{\prime \prime} \mathrm{S} 60^{\circ} 04^{\prime} 53^{\prime \prime} \mathrm{W}$, mixed light trap, 17-24. VI.2004, F.F. Xavier Fo \& L. Aquino colls., T.M. Almeida, 2013 det.; 1 female, pinned, Iranduba municipality, Catalão lake, $03^{\circ} 15^{\prime} \mathrm{S} 59^{\circ} 54^{\prime} \mathrm{W}$, light trap, 03-07.XII.2001, A.M.O. Pes, C.A. Azevedo, N. Hamada \& J.L. Nessimian colls., T.M. Almeida, 2013 det.; 1 male, ethyl alcohol 70\%, Itacoatiara municipality, Trindade island, $03^{\circ} 19^{\prime} 29.2^{\prime \prime} \mathrm{S} 58^{\circ} 44^{\prime} 32.7^{\prime \prime} \mathrm{W}$, A17L, 22.IX.2003, P. de Marco \& N. Ferreira-Jr. colls., V.P. Alecrim det.; 1 male, pinned, Itacoatiara municipality, 21.IV.1980, 0020921, T.M. Almeida, 2013 det.; 1 male, pinned, Itacoatiara municipality, Mamoud Amed bridge, AM 010, 15-16.V.1991, C.S. Motta, F.S.A. Peralta, B.R. Teles \& R. Andreazze colls., 0021019, T.M. Almeida, 2013 det.; 1 male, pinned, Manacapuru municipality, $03^{\circ} 36^{\prime} 37^{\prime \prime S}$ $61^{\circ} 17^{\prime} 56^{\prime \prime}$ W, light trap, 29.Il.2004, F.F. Xavier Fo coll., T.M. Almeida, 2013 det.; 1 female, Manaus municipality, AM 010 km 134, 10.VIII.1968, 2969, 0021059, T.M. Almeida, 2013 det.; 1 female, pinned, Manaus municipality, VIII.1956, 0020907, T.M. Almeida, 2013 det.; 1 male, pinned, Manaus municipality, 01.VIII.1956, N.L. Cerqueira coll., 0020903, T.M. Almeida, 2013 det. 1 male, ethyl alcohol 70\%, Pará, Almeirim municipality, $01^{\circ} 28^{\prime} 48.2^{\prime \prime} \mathrm{S} 52^{\circ} 28^{\prime} 31.4^{\prime \prime} \mathrm{W}, \mathrm{A} 26 \mathrm{~L}$, 07.X.2003, P. de Marco \& N. Ferreira-Jr., V.P. Alecrim det. 
1 female, ethyl alcohol 70\%, Roraima, Amajari municipality, Maracá Ecological Station, near Uraricoera river, 23.IX.1987, team Nhanhame colls., M.E. Lanzer-de-Souza, 1987 det.

Notes: This is the first record of $B$. dentatum to Roraima state (Brazil)

\section{Belostoma elongatum Montandon, 1908} (Figs. 1F, 5A)

Known distribution: ARGENTINA: Buenos Aires (De Carlo, 1930), Catamarca (De Carlo, 1930), Chaco (De Carlo, 1930), Corrientes (De Carlo, 1930), Entre Ríos (De Carlo, 1930), Mendoza (Lauck, 1962), Misiones (Lauck, 1962), Salta (Ribeiro, 2007), Santa Fe (Lauck, 1962), Santiago del Estero (Lauck, 1962). BOLIVIA: Roboré (Lauck, 1962), Santa Cruz (Lauck, 1962), Pando (Lauck, 1962). BRAZIL: Mato Grosso (Heckman, 1998), Mato Grosso do Sul (Ribeiro, 2007), Minas Gerais (Ribeiro, 2007), Piauí (Ribeiro, 2007), Rio Grande do Sul (Lanzer, 1976), Rondônia (Ribeiro, 2007). PARAGUAY: Alto Paraguay (Ribeiro, 2007), Asunción (Montandon, 1908), Guairá (Lauck, 1962), Paraguarí (Lauck, 1962). PERU: Junín (Lauck, 1962). URUGUAY: (De Carlo, 1930), Artigas (Ruffinelli \& Pirán, 1959), Canelones (Ruffinelli \& Pirán, 1959), Montevideo (Ruffinelli \& Pirán, 1959), Rivera (Ruffinelli \& Pirán, 1959), San José (Lauck, 1962), Treinta y Tres (Ruffinelli \& Pirán, 1959).

Material examined: 2 males and 2 females, pinned, BRAZIL, Mato Grosso, Poconé municipality, Ipiranga farm, $16^{\circ} 23^{\prime} 06^{\prime \prime} \mathrm{S} 56^{\circ} 35^{\prime} 28^{\prime \prime} \mathrm{W}$, Malaise trap, 02.V.2006, J.A. Rafael \& F.F. Xavier Fo colls., T.M. Almeida, 2013 det.; 5 males and 2 females, pinned, Chapada dos Guimarães municipality, $15^{\circ} 21^{\prime} 23^{\prime \prime} \mathrm{S} 55^{\circ} 49^{\prime} 48^{\prime \prime} \mathrm{W}$, light trap, 05.V.2006, J.A. Rafael \& F.F. Xavier Fo colls., T.M. Almeida, 2013 det. 2 females, pinned, Paraná, Tibagi municipality, Guartelá State Park, light trap, 10.XI.2007, J.A. Rafael \& P. Grossi colls., T.M. Almeida, 2013 det.; 1 female, pinned, Londrina municipality, 13.X.2012, F. Carduco coll., T.M. Almeida, 2013 det.

Notes: This is the first record of B. elongatum to Paraná state (Brazil).

\section{Belostoma foveolatum (Mayr, 1863) (Figs. 1G, 5B)}

Known distribution: ARGENTINA: Buenos Aires (Berg, 1879), Misiones (Digiani, 2002), Tucumán (Berg, 1879), unknow Province (De Carlo, 1938). BRAZIL: Ceará (Ribeiro, 2000), Mato Grosso (Dias-Silva et al., 2010), Mato Grosso do Sul (Ribeiro, 2000), Minas Gerais (De Carlo, 1938), Pará (Lauck, 1962), Rio de Janeiro (De Carlo, 1938), São Paulo (De Carlo, 1938). FRENCH GUYANA: Cayenne (Mayr, 1863). PARAGUAY: Alto Paraguay (Montandon, 1895), unknow Department (De Carlo, 1938).
Material examined: 3 males and 1 female, pinned, BRAZIL, Ceará, Viçosa do Ceará municipality, 03.VI.2011, T.M. Almeida, 2017 det. 1 male, pinned, Pará, 0020990, T.M. Almeida, 2017 det.

\section{Belostoma guianae Lauck, 1962 (Figs. 1H, 5A)}

Known distribution: GUYANA: Cuyuni-Mazaruni (Lauck, 1962), Demerara-Mahaica (Lauck, 1962), East Berbice-Corentyne (Lauck, 1962), Essequibo Islands-West Demerara (Lauck, 1962), Upper Takutu-Upper Essequibo (Nieser, 1975). SURINAM: Surinam (Nieser, 1975).

Material examined: 3 males and 5 females, ethyl alcohol 70\%, BRAZIL, Roraima, Amajari municipality, Maracá Ecological Station, near Uraricoera river, light trap, 21-23. IX.1987, team Nhamenhame colls., M.E. Lanzer-de-Souza, 1987 det.

Notes: This is the first record of B. guianae to Brazil (Roraima).

\section{Belostoma harrisi Lauck, 1962}

(Figs. 1I, 5B)

Known distribution: ARGENTINA: unknow Province (Ribeiro et al., 2017). BRAZIL: Amazonas (De Carlo, 1966), Pará (Lauck, 1962), Roraima (Ribeiro et al., 2017). GUYANA: Demerara-Mahaica (Lauck, 1962), unknow Region (Nieser, 1975). PERU: unknow Region (Ribeiro et al., 2017). SURINAM: Commewijne (Nieser, 1975), Marowijne (Nieser, 1975), Nickerie (Nieser, 1975), Saramacca (Nieser, 1975), unknow District (Lauck, 1962). VENEZUELA: unknow State (Ribeiro et al., 2017).

Material examined: 1 male, ethyl alcohol 70\%, BRAZIL, Amazonas, Careiro da Várzea municipality, Purupuru lake, T.M. Almeida, 2014 det.; 1 male, ethyl alcohol 70\%, Careiro da Várzea municipality, Careiro village, $9 \mathrm{~km}$ of East, $03^{\circ} 11^{\prime} 27^{\prime \prime S} 59^{\circ} 50^{\prime} 36^{\prime \prime} \mathrm{W}, 07-08 . I X .2013$, N.G. Cipola coll., T.M. Almeida det.; 1 male, ethyl alcohol 70\%, Iranduba municipality, T.M. Almeida, 2013 det.; 1 male, pinned, Iranduba municipality, Catalão lake, $03^{\circ} 15^{\prime} \mathrm{S}$ 595' W, 03-07.XII.2001, T.M. Almeida, 2013 det.; 1 female, ethyl alcohol 70\%, Solimões river, between Coari and Codajás municipalities, 0241'09.2"S 66²5'31.0"W, 14.IX.2003, N. Hamada \& J.L. Nessimian colls., J.R.I. Ribeiro det.; 1 female, pinned, Itacoatiara municipality, 21.IV.1980, 131, 0020925, T.M. Almeida, 2017 det. 1 male, pinned, Pará, near Mojuí dos Campos municipality, Curuá-una river, 01.III.1982, R.E. Best., 0020875, T.M. Almeida, 2013 det. 1 male, pinned, Roraima, Amajari municipality, Uraricoera river, Maracá Ecological Station, light trap, 0020997, 21-30.XI.1987, J.A. Rafael et al. colls., T.M. Almeida, 2013 det.; 1 male, pinned, Amajari municipality, Uraricoera river, Maracá Ecological Station, light trap, 0020923, 21-30.XI.1987, J.A. Rafael et al. colls., T.M. 
Almeida, 2013 det.; 1 female, pinned, Amajari municipality, Uraricoera river, Maracá Ecological Station, mixed light trap, 16-17.III.1988, 002906, T.M. Almeida, 2013 det.; 1 female, pinned, Amajari municipality, Uraricoera river, Maracá Ecological Station, mixed light trap, 21-22. III.1988, 0020905, T.M. Almeida, 2013 det.

\section{Denticolle group sensu Estévez \& Polhemus (2001)}

Diagnose: Ventral diverticulum flat, arms of phallobase longer than posterior portion of phalobase; pilosity well developed, covering a moderately wide band of the connexivum.

\section{Belostoma amazonum Estévez \& Polhemus, 2001 (Figs. 1J, 6A)}

Known distribution: BRAZIL: Amazonas (Estévez \& Polhemus, 2001), Bahia (Estévez \& Polhemus, 2001), Santa Catarina (Estévez \& Polhemus, 2001). VENEZUELA: Calabozo (Estévez \& Polhemus, 2001).

Material examined: 1 female, ethyl alcohol 70\%, BRAZIL, Amazonas, Iranduba municipality, Manoel Urbano highway, km 07, Janauarí lake, km 8.5, 03¹3'47.4"S 6006'14.7"W, 12.XII.2012, T.M. Almeida, G.A. Gusmão, D. Colpani,G.P.S.Dantas\&J.S.Oliveiracolls.,T.M.Almeida,2017 det.; 8 males and 13 females, ethyl alcohol 70\%, Iranduba municipality, Manoel Urbano highway, km 07, Janauarí

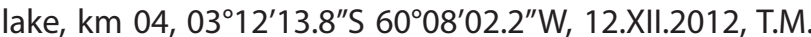
Almeida, G.P.S. Dantas, G.A. Gusmão, D. Colpani \& J.S. Oliveira colls., T.M. Almeida, 2017 det.; 2 males, ethyl alcohol $70 \%$, Iranduba municipality, Marchantaria island, 21.VIII.2012. T.M. Almeida, G.A. Gusmão, D. Colpani \& G.P.S. Dantas colls., T.M. Almeida, 2017 det.; 2 males, ethyl alcohol 70\%, Itacoatiara municipality, aquatic insect net, 03.XI.2012, C.A. Azevêdo, E.S.Pereira, P. Reis, R.L. FerreiraKeppler colls., T.M. Almeida, 2017 det.; 4 males and 3 females, ethyl alcohol 70\%, Manaus municipality, Bosque da Ciência - INPA campus I, Amazônico lake, aquatic insect net, 13.VIII.2012, T.M. Almeida, G.A. Gusmão, D. Colpani \& C.R.S. Neto colls., T.M. Almeida, 2017 det.; 8 males and 3 females, ethyl alcohol 70\%, Manaus municipality, 19.XI.2012, T.M. Almeida, G.A. Gusmão, D. Colpani \& D. Costa colls., T.M. Almeida, 2017 det.; 3 males and 2 females, ethyl alcohol 70\%, Manaus municipality, Green Park Residencial, in pool, 20.VIII.2001, T.M. Almeida, 2017 det.; 1 female, ethyl alcohol 70\%, Manaus municipality, Puraquequara lake, $03^{\circ} 03^{\prime} 48.9^{\prime \prime} \mathrm{S} 59^{\circ} 50^{\prime} 46.3^{\prime \prime} \mathrm{W}$ 13.XII.2012, T.M. Almeida, G.A. Gusmão, D. Colpani \& J.S. Oliveira colls., T.M. Almeida, 2018 det.; 2 males and 1 female, ethyl alcohol 70\%, Manaus municipality, Centro de Projetos e Estudos Ambientais do Amazonas - Cepeam/ Soka Gakkai, 0306'44.1"S 5954'22.7"W, aquatic insect net, 11.XII.2012, T.M. Almeida, G.P.S. Dantas, G.A. Gusmão, D. Colpani \& J.S. Oliveira colls., T.M. Almeida, 2017 det.; 1 female, ethyl alcohol 70\%, near Manaus municipality, BR 174, km 60, 04-07.V.2007, C.A. Nogueira coll., C.A.
Nogueira, 2007 det.; 1 male, pinned, near Manaus municipality, estrada AM 010, km 64, 29.VII.1970, A. Faustino coll., 3013, 0021040, J.R.I. Ribeiro, 2001 det.; 1 male, ethyl alcohol 70\%, AM 010, near Manaus municipality, Onça stream, T.M. Almeida, 2017 det.; 1 female, ethyl alcohol 70\%, near Manaus municipality, Pretinho stream, 29.IX.2014, T.M. Almeida, 2018 det.; 7 males and 11 females, ethyl alcohol 70\%, near Manaus municipality, BR 174, km 21, 0247'31.8"S 6002'04.6"W, 10.XII.2012, T.M. Almeida, G.A. Gusmão, D. Colpani \& D. Costa colls., T.M. Almeida, 2017 det. 1 male and 1 female, ethyl alcohol $70 \%$, Presidente Figueiredo municipality, BR 174, km 121, $01^{\circ} 55^{\prime} 50.2^{\prime \prime} \mathrm{S} 60^{\circ} 03^{\prime} 02.0^{\prime \prime} \mathrm{W}, 10 . X I I .2012$, T.M. Almeida, G.A.
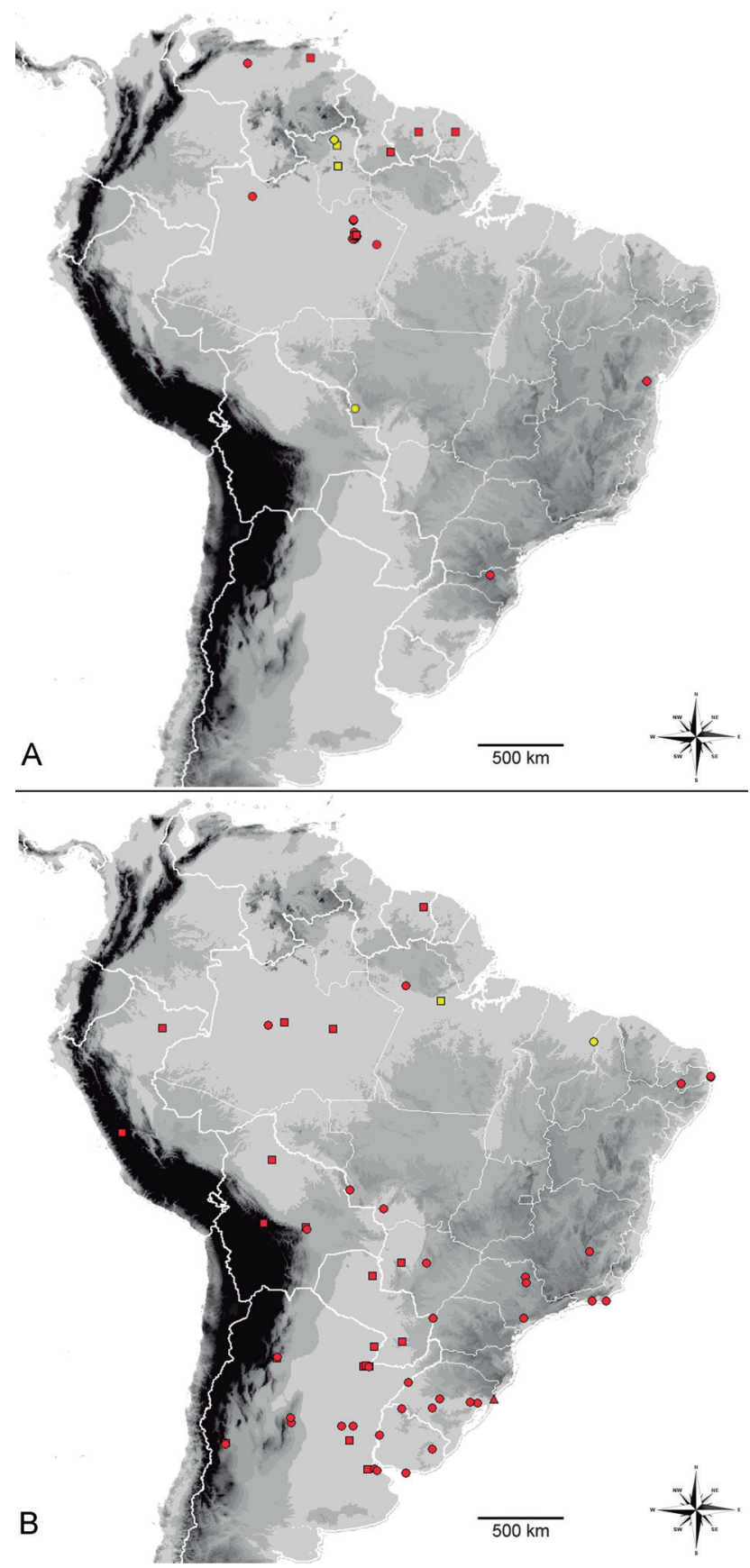

Figure 6. Distribution of Belostomatidae in South America. Red symbol: known records, yellow symbol: new records. $(A)=$ Belostoma amazonum (circle), B. denticolle (square). (B) = B. gestroi (square), B. dilatatum (circle), B. ripicolum (triangle). 
Gusmão, D. Colpani, G.P.S. Dantas \& J.S. Oliveira colls., T.M. Almeida, 2017 det.; 3 males and 7 females, ethyl alcohol 70\%, Presidente Figueiredo municipality, Santa Cruz stream, $01^{\circ} 54^{\prime} 09.0^{\prime \prime S} 60^{\circ} 03^{\prime} 48.3^{\prime \prime} \mathrm{W}, 10 . \mathrm{XII.2012,} \mathrm{T.M.}$ Almeida, G.A. Gusmão, D. Colpani, G.P.S. Dantas \& J.S. Oliveira colls., T.M. Almeida, 2017 det.; 2 males and 1 female, ethyl alcohol 70\%, Presidente Figueiredo municipality, AM 240, km 26, Pantera stream, 17.VIII.2011, T.M. Almeida, 2018 det.; 1 male, ethyl alcohol 70\%, São Gabriel da Cachoeira municipality, near airport, 07.VIII.2010, R. Ferreira-Keppler coll., T.M. Almeida, 2017 det.; 1 female, ethyl alcohol 70\%, São Gabriel da Cachoeira municipality, Morro dos Seis Lagos Biological Reserve, 09.X.1990, team Neblina colls., T.M. Almeida, 2018 det. 1 male, ethyl alcohol, Mato Grosso, Vila Bela da Santíssima Trindade municipality, Guaporé river, 01.X.1984, T.M. Almeida, 2018 det. 1 male with eggs, ethyl alcohol 70\%, Roraima, Amajari municipality, Amajari river, 27.VIII.1987, 1675, team Ericó colls., T.M. Almeida, 2013 det.

Notes: This is the first record of B. amazonum to Mato Grosso and Roraima states (Brazil).

\section{Belostoma denticolle Montandon, 1903 (Figs. 1K, 6A)}

Known distribution: BRAZIL: Amazonas (Ribeiro et al., 2018). FRENCH GUYANA: Cayenne (Montandon, 1903a). GUYANA: East Berbice-Corentyne (Estévez \& Polhemus, 2001). VENEZUELA: Monagas (Estévez \& Polhemus, 2001). SURINAME (Montandon, 1903a).

Material examined: 1 male, pinned, BRAZIL, Amazonas, near Manaus municipality, AM 010, km 45, 25.IV.1982, 0020937, E.F. Ribeiro coll., J.R.I. Ribeiro, 2004 det.; 1 male, ethyl alcohol 70\%, Iranduba municipality, Manoel Urbano highway, AM 010, km 08, 23.VIII.2012, G.A. Gusmão et al. colls., T.M. Almeida, 2013 det. 1 male, pinned, Roraima, Caracaraí municipality, 13.V.1977, N.D. Penny coll., 0021017, J.R.I. Ribeiro, 2004 det.; 1 female, ethyl alcohol 70\%, Amajari municipality, Uraricoera River, 23.X.1987, team Granfinale colls., T.M. Almeida, 2018 det.

Notes: This is the first record of $B$. denticolle to Roraima state (Brazil).

\section{Dilatatum group sensu Lauck (1962)}

Diagnose: Body expanded laterally, ovate species reaching about $50 \mathrm{~mm}$ in length; eye acutely triangular, outer margin nearly straight.

\section{Belostoma dilatatum (Dufour, 1863) (Figs. 1L, 6B)}

Known distribution: ARGENTINA: Buenos Aires (De Carlo, 1938), Córdoba (Lauck, 1963), Corrientes (De Carlo,
1938), Entre Ríos (De Carlo, 1938), La Granja, Mendoza (Lauck, 1963), Quilmes (Schnack, 1976), San Miguel de Tucumán, Santa Fe (Lauck, 1963), Santo Tomé (De Carlo, 1938), Tucumán (Lauck, 1963). BOLIVIA: Santa Cruz (Lauck, 1963). BRAZIL: Amazonas (Ribeiro et al., 2018), Mato Grosso (Ribeiro, 2005), Mato Grosso do Sul, Minas Gerais (Ribeiro, 2005), Pará (Ribeiro, 2005), Paraíba (Ribeiro, 2005), Rio de Janeiro (Ribeiro, 2005), Rio Grande do Sul (Lanzer, 1976), São Paulo (Ribeiro, 2005). PARAGUAY: Guairá (Lauck, 1963). URUGUAY: Artigas (Ruffinelli \& Pirán, 1959), Montevideo (Ruffinelli \& Pirán, 1959), Paysandú (Lauck, 1963), Treinta y Tres (Ruffinelli \& Pirán, 1959).

Material examined: 1 male, pinned, BRAZIL, Paraíba, João Pessoa municipality, VII.1977, 0020868, V. PyDaniel coll., T.M. Almeida, 2017 det. 1 male, pinned, Maranhão, Caxias municipality, in headwater, 04³9'32"S $43^{\circ} 03^{\prime} 51^{\prime \prime}$, light trap, 19.V.2007, J.A. Rafael, F.F. Xavier Fo \& F.L. Oliveira colls., T.M. Almeida, 2017 det. 1 male, ethyl alcohol 70\%, Mato Grosso, Vila Bela da Santíssima Trindade municipality, Guaporé river, 1810, 01.X.1984, team Rosé colls., T.M. Almeida, 2017 det. 1 female, ethyl alcohol 70\%, Pará, Trombetas river, 11.III.1986, team Apoidea, T.M. Almeida, 2017 det.; 1 female, ethyl alcohol 70\%, Boa Sorte stream, 22.IV.1989, U. Barbosa coll., T.M. Almeida, 2017 det.

Notes: This is the first record of B. dilatatum to Maranhão state (Brazil).

\section{Belostoma ripicolum Lanzer-de-Souza, 1992 junior synomy of $B$. dilatatum (Figs. 2A, 6B)}

Known distribution: BRAZIL: Rio Grande do Sul. Capão da Canoa (Lanzer-de-Souza, 1992).

Type Material examined: 1 male paratype, pinned, BRAZIL, Rio Grande do Sul, Capão da Canoa municipality, Lagoa dos Quadros, M.E.L. de Souza, 1992 coll., 135358, M.E.L. de Souza, 1992 det.

Notes: This species was described by Lanzer-de-Souza (1992) as B. ripicola, it is an incorrect original spelling because Belostoma is a neuter noun, not a feminine one. Thus, Ribeiro (2007) changed the name of this species and synonymized.

\section{Belostoma gestroi Montandon, 1900 (Figs. 2B, 6B)}

Known distribution: ARGENTINA: Chaco (Ribeiro, 2004), Formosa (Ribeiro, 2004), Buenos Aires, Corrientes, Entre Ríos, Mendoza, Resistencia, Tucumán (Contartese \& Bachmann, 1987; De Carlo, 1930, 1938; Lauck, 1963;Nieser, 1975; Schnack, 1976). BOLIVIA: Beni, Cochabamba, Santa Cruz (De Carlo, 1930, 1938; Lauck, 1963; Nieser, 
1975). BRAZIL: Amazonas (Ribeiro, 2004; Lauck, 1963), Mato Grosso do Sul (Ribeiro, 2004), Mato Grosso (Lauck, 1963). PARAGUAY: Coquelet (Montandon, 1900), Guairá (Lauck, 1963). PERU: Junín, Loreto (Lauck, 1963; Nieser, 1975; Schnack, 1976). SURINAM: Brokopondo (Nieser, 1975).

Material examined: 1 male, pinned, BRAZIL, Amazonas, near Tefé municipality, Tefé lake, near Solimões river, 10.XII.1976, E. Faustino coll., 0020901, J.R.I. Ribeiro, 1998 det. 1 male, ethyl alcohol 70\%, Pará, Prainha municipal-

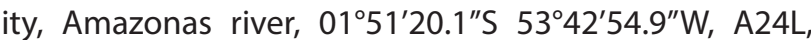
04.X.2003, P. de Marco \& N. Ferreira-Jr. colls., V.P. Alecrim det.

Notes: This is the first record of $B$. gestroi to Pará state (Brazil).

\section{Discretum group sensu Lauck (1963)}

Diagnose: Scutellum does not reach the nodal line; eye subtriangular and beak with segment I conspicuously shorter than II.

\section{Belostoma discretum Montandon, 1903} (Figs. 2C, 7A)

Known distribution: ARGENTINA: Chaco (De Carlo, 1938), Corrientes (Montandon, 1903b), Entre Rios (De Carlo, 1930), Santa Fé (Lauck, 1963). BOLIVIA: Beni (Lauck, 1963), Reyes (Lauck, 1963). BRAZIL: Acre (Ribeiro, 2005), Amazonas (De Carlo, 1938), Goiás (Menke \& Lauck, 1962), Mato Grosso (De Carlo, 1930), Mato Grosso do Sul (Ribeiro, 2005), Pará (Nieser, 1975), Rio de Janeiro (Ribeiro, 2005), Rondônia (Ribeiro, 2005), São Paulo (Ribeiro, 2007), Tocantins (Ribeiro et al., 2018). PARAGUAY: unknow Departament (Montandon, 1903b). PERU: Amazonas (Lauck, 1963), Junín (Lauck, 1963), Loreto (Lauck, 1963), Marcapata (Ribeiro, 2007), Pucallpa (Lauck, 1963), Trapiche river (Lauck, 1963), Ucayali river (Lauck, 1963).

Material examined: 1 male, pinned, BRAZIL, Amazonas, 06.X.1977, B.C. Ratcliffe coll., 1998, 0021011, J.R.I. Ribeiro det.; 1 male, pinned, 12.VIII.1977, B.C. Ratcliffe coll., 1998, 0020930, J.R.I. Ribeiro det.; 1 male, pinned, Amanã lake, 17.IX.1979, R. Best coll., 0020992, J.R.I. Ribeiro, 1998 det.; 1 male, pinned, Amanã lake, 10.IX.1979, R. Best coll., J.R.I. Ribeiro, 1998 det.; 2 males, pinned, Nhamundá river 013'11"S 573'32"W, light trap, 17-20.V.2008, J.A. Rafael et al. colls., T.M. Almeida, 2017 det.; 1 male, pinned, ZF-3 Reserve, 19.Il.1988, A.V.V. Silva coll., 1202, 0020913, J.R.I. Ribeiro, 1998 det.; 1 male, pinned, Purus river, 24.X.1979, J.C. Campbell coll., 0021011 , J.R.I. Ribeiro, 1998 det.; 1 female, pinned, ZF-3 Reserve, 19.IV.1988, A.V.V. Silva coll., 0020912, T.M. Almeida, 2013 det.; 1 male and 3 females, ethyl alcohol 70\%, Grande lake, near Purus river, 30-31.XI.1986, F. Peralta coll., 1860, T.M. Almeida, 2013 det.; 1 male, ethyl alcohol 70\%, Albano lake, near

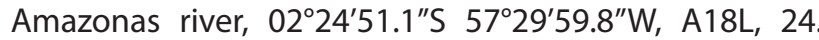
IX.2003, P. de Marco \& N. Ferreira-Jr., V.P. Alecrim det.; 1 male, pinned, Urucuri-Codajás municipalities, XII.1964, E. Faustino coll., 3013, 0021044, J.R.I. Ribeiro, 1998 det.; 1 male, pinned, Urucuri-Codajás municipalities, XII.1965, E. Faustino coll., 0021048, 2552, J.R.I. Ribeiro, 1998 det.; 1 male, pinned, Urucuri-Codajás municipalities, XII.1964, E. Faustino coll., 002552, J.R.I. Ribeiro, 1998 det.; 1 male, pinned, Careiro Castanho municipality, Castanho lake, 02-18.VI.1976, J.M. Santos coll., 3013, 0021044, J.R.I. Ribeiro, 1998 det.; 1 male, pinned, Urucuri-Codajás municipalities, 19.X.1964, E. Faustino coll., 0021054, 2552, J.R.I. Ribeiro, 1998 det.; 1 male, pinned, Urucuri-Codajás

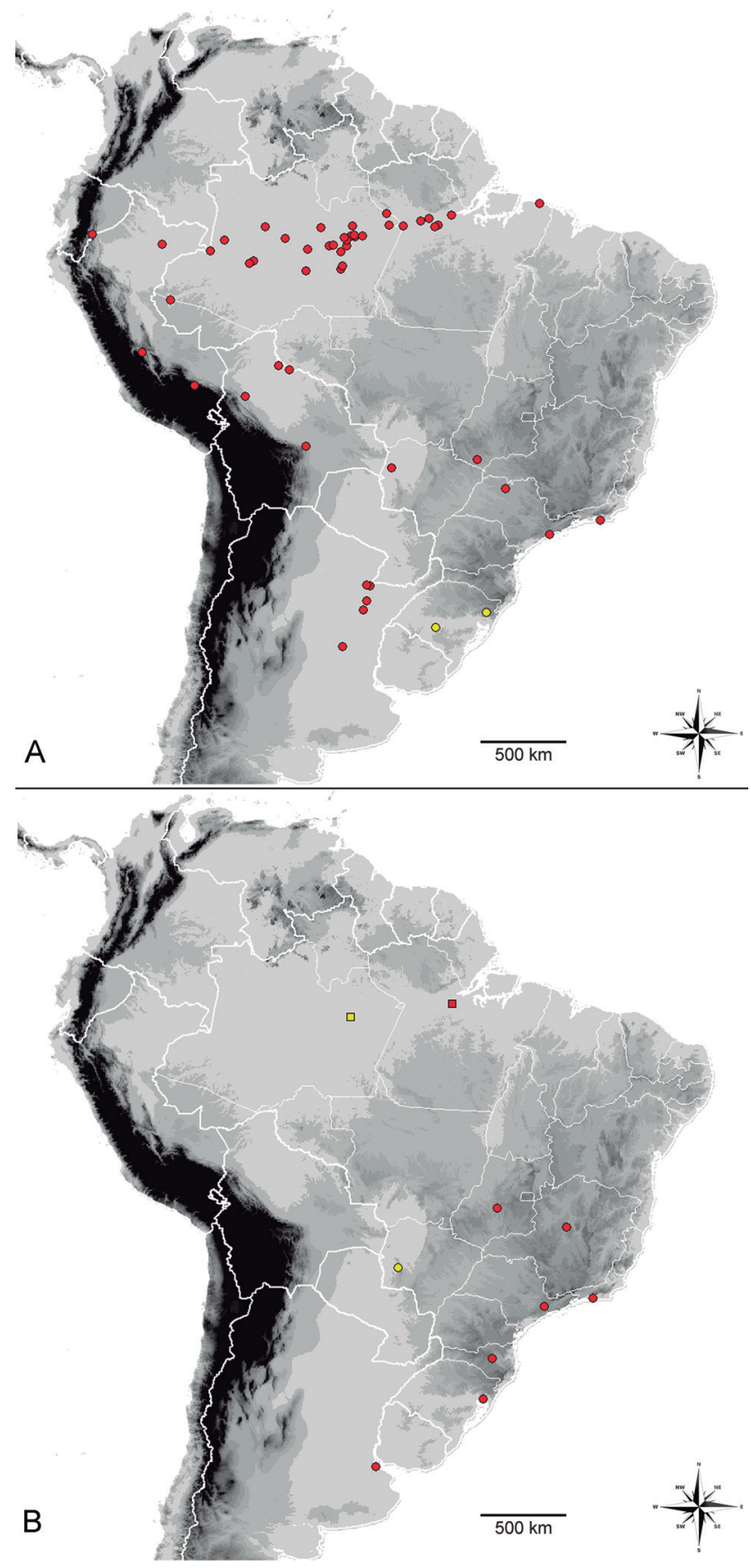

Figure 7. Distribution of Belostomatidae in South America. Red symbol: known records, yellow symbol: new records. $(A)=$ Belostoma discretum. $(B)=$ B. carajaensis (square), B. candidulum (circle). 
municipalities, XII.1964, E. Faustino coll., 002615, J.R.I. Ribeiro, 1998 det.; 1 female, pinned, Solimões river, XII.1963, E. Faustino coll., 3013, 0021044, J.R.I. Ribeiro, 1998 det.; 1 male, pinned, Solimões river, 20.III.1979, A.M. Penny coll., J.R.I. Ribeiro, 1998 det.; 1 male, pinned, Solimões river, 04.XII.1962, several collectors, 0021055, 2482, J.R.I. Ribeiro, 1998 det.; 1 male, pinned, Solimões river, 16.VI.1977, B.C. Ratcliffe coll., 0021008, J.R.I. Ribeiro, 1998 det.; 1 male, pinned, Solimões river, 16.VI.1977, B.C. Ratcliffe coll., 0021001, J.R.I. Ribeiro, 1998 det.; 1 female, pinned, Anorí municipality, 0350'33"S 61³9'48"W, 19. IX.2006, F.F. Xavier Fo coll., T.M. Almeida, 2017 det.; 1 female, pinned, near Amaturá municipality, Amaturá stream, R.S., 18.IX.1961, E. Faustino coll., 2298, 0020928, T.M. Almeida, 2013 det.; 1 male, pinned, Beruri municipality, Purus river, mixed light trap, 30.IX-09.X.2002, F.F. Xavier Fo \& U.C. Barbosa colls., T.M. Almeida, 2013 det.; 1 male, ethyl alcohol 70\%, Caapiranga municipality, Mureru lake, near Amazonas river, A23L, 02.X.2003, P. de Marco \& N. Ferreira-Jr. colls., V.P. Alecrim det.; 1 male and 1 female, ethyl alcohol 70\%, Caapiranga municipality, Mureru lake, near Amazonas river, A23L, 02.X.2003, P. de Marco \& N. Ferreira-Jr. colls., V.P. Alecrim det.; 1 female, ethyl alcohol 70\%, Caapiranga municipality, Mureru lake, near Amazonas River, A23L, 02.X.2003, P. de Marco \& N. Ferreira-Jr. colls., V.P. Alecrim det.; 1 male, pinned, Carauari municipality, 04.II.1988, 0029915, 045, M.F. Vieira \& J.R.I. Ribeiro, 1998 det.; 4 males and 4 females, pinned, Carauari municipality, $05^{\circ} 00^{\prime} 00^{\prime \prime} \mathrm{S} 67^{\circ} 10^{\prime} 03^{\prime \prime} \mathrm{W}$, mixed light trap, VII.2005, A. Henriques \& F.F. Xavier Fo colls., J.R.I. Ribeiro, 1998 det.; 1 male and 1 female, ethyl alcohol 70\%, Careiro da Várzea municipality, Purupuru lake, km 35, BR 319, aquatic insect net, 08.VIII.2012, N. Hamada, J.O. Silva, N. Zampiva \& N. Hidalgo colls., T.M. Almeida, 2013 det.; 2 males and 8 females, ethyl alcohol 70\%, Careiro da Várzea municipality, Purupuru lake, 08.VIII.2012, N. Hamada, J.O. Silva, N. Zampiva \& N. Hidalgo colls., T.M. Almeida, 2013 det.; 1 female, pinned, Careiro da Várzea municipality, Careiro island, 29.IX.1959, Sioli \& Sattler colls., 0020895, H.H, Weber, 1966 det.; 1 male, pinned, Careiro da Várzea municipality, 14.III.1961, E.J. Fittkau coll., 126, 0020899, H.H. Weber, 1966 det.; 1 female, pinned, Careiro Castanho municipality, 03 $50^{\circ} 42^{\prime \prime} \mathrm{S}$ 60'26'03"W, canopy light trap, 21:00-00:00 h, 04-05. XI.2010, J.A. Rafael, D.M. Takiya, F.F. Xavier Fo, M.J. Holanda \& D.M.M. Mendes colls., T.M. Almeida, 2018 det.; 1 female, pinned, Careiro da Várzea municipality, 03 $50^{\circ} 42^{\prime \prime} S$ 60²6'03"W, canopy light trap, 18:00-21:00 h, 04-05. XI.2010, J.A. Rafael, D.M. Takiya, F.F. Xavier Fo, M.J. Holanda \& D.M.M. Mendes colls., T.M. Almeida, 2018 det.; 1 female, pinned, Careiro da Várzea municipality, BR 319, km 180, 04'16'28"S 6050'45"W, 05.XI.2010, mobile light trap, 21:00-00:00 h, J.A. Rafael, D.M. Takiya, F.F. Xavier Fo, M.J. Holanda \& D.M.M. Mendes colls., T.M. Almeida, 2018 det.; 1 male, pinned, Coari municipality, 20.VIII.1979, Mag. Nisson \& V. Py-Daniel colls., 0020994, J.R.I. Ribeiro, 1998 det.; 1 male, pinned, Coari municipality, 20.VIII.1979, Mag. Nisson \& V. Py-Daniel colls., 0020994, J.R.I. Ribeiro, 1998 det.; 1 male, pinned, Fonte Boa municipality, 02³2'27"S $66^{\circ} 04^{\prime} 08^{\prime \prime}$, light trap, 23.IX.2005, J.A. Rafael \& F.F. Xavier
Fo colls., T.M. Almeida, 2013 det.; 1 male, pinned, Iranduba municipality, Marchantaria island, 26.III.1976, Epujino coll., J.R.I. Ribeiro, 1998 det.; 3 males and 1 female, ethyl alcohol 70\%, Iranduba municipality, Marchantaria island, 21.VIII.2012, T.M. Almeida, G.A. Gusmão, D. Colpani, G.P.S. Dantas colls., T.M. Almeida, 2013 det.; 1 male and 1 female, ethyl alcohol 80\%, Iranduba municipality, Marchantaria island, aquatic insect net, 21.VIII.2012, T.M. Almeida, G.A. Gusmão, D. Colpani \& G.P.S. Dantas colls., T.M. Almeida, 2013 det.; 4 males, ethyl alcohol 70\%, Iranduba municipality, Manoel Urbano highway, AM 070, km 08, 23.VIII.2012. G.A. Gusmão et al. colls., T.M. Almeida, 2013 det.; 1 female, pinned, Iranduba municipality, Catalão lake, $03^{\circ} 15^{\prime} \mathrm{S} 59^{\circ} 54^{\prime} \mathrm{W}$, light trap, 03-07.XII.2001, A.M.O. Pes, C.A. Azevedo, N. Hamada \& J.L. Nessimian colls., T.M. Almeida, 2013 det.; 26 males and 11 females, ethyl alcohol 80\%, Iranduba municipality, Manoel Urbano highway, km 07, Janauari lake, km 4, 0312'13.8"S $60^{\circ} 08^{\prime} 02.2^{\prime \prime} \mathrm{W}, 12 . X I 1.2012$, T.M. Almeida, G.P.S. Dantas, G.A. Gusmão, D. Colpani \& J.S. Oliveira colls., T.M. Almeida, 2013 det.; 1 female, pinned, Iranduba municipality, Marchantaria island, 26.III.1976, I.S. Gorayeb coll., 0020927, J.R.I. Ribeiro, 1998 det.; 1 female, pinned, Itacoatiara municipality, PoxTE Mamous AMD, Urubu river, AM 010, km 247, 18-19.XI.1990, C.S. Motta, F.A. Peralta, Z.R. Teles colls., 0021013, J.R.I. Ribeiro, 1998 det.; 3 males, pinned, Iranduba municipality, Urubu river, AM 010, km 247, 18-19.XI.1990, C.S. Motta, F.A. Peralta, B.R. Teles \& R. Andreazze colls., 0021020, T.M. Almeida, 2013 det.; 1 male, pinned, Iranduba municipality, Aruanã farm, AM 010 km 215, light trap, 22-23.VII.1990, C.S. Motta, F.A. Peralta, N. Hamada \& Andreazze colls., 0021015, T.M. Almeida, 2013 det.; 1 male, pinned, Iranduba municipality, 22-23.VIII.1990, C.S. Motta, F.A. Peralta, B. Ronchi Teles colls., 0021012, T.M. Almeida, 2013 det.; 1 female, ethyl alcohol 70\%, Iranduba municipality, N.S. do Perpétuo Socorro, Amazonas river, Traíra lake, 0309'35.9"S 59¹9'37.8"W, A16L, 21.IX.2003, P. de Marco \& N. Ferreira-Jr. colls., V.P. Alecrim det.; 1 male, ethyl alcohol 70\%, Iranduba municipality, Amazonas river, Trindade island, 0319'29.2"S 5844'32.7"W, A17L, 22.IX.2003, P. de Marco \& N. Ferreira-Jr. colls., V.P. Alecrim det.; 2 males, pinned, Manaquiri municipality, 12-13.IV.1996, J.F.L. Moura coll., T.M. Almeida, 2013 det.; 1 male, pinned, Manaquiri municipality, Janauacá lake, 16.VI.1977, M. Serrano coll., 0020873, J.R.I. Ribeiro, 1998 det.; 1 male, pinned, Manaus municipality, 18.VIII.1964, E. Faustino coll., 2703, 0021051, J.R.I. Ribeiro, 1998 det.; 1 male, pinned, Manaus municipality, Aleixo district, 04.II.1988, M.F. Vieira coll., 0020915, J.R.I. Ribeiro, 1998 det.; 1 male, ethyl alcohol 70\%, Manaus municipality, UFAM campus, ESALQ, Blb, 27-28.IX.1982., T.M. Almeida, 2013 det.; 3 males (1 with eggs) and 7 females, ethyl alcohol 70\%, Manaus municipality, Bosque da Ciência - INPA campus I, Amazônico lake, aquatic insect net, 19.XI.2012, T.M. Almeida, G.A. Gusmão, D. Colpani \& D. Costa colls., T.M. Almeida, 2013 det.; 6 males, ethyl alcohol 70\%, Manaus municipality, aquatic insect net, 13.VIII.2012, T.M. Almeida, G.A. Gusmão, D. Colpani \& C.R.S. Neto colls., T.M. Almeida, 2013 det.; 1 male, pinned, Manaus municipality, campus INPA, Aleixo district, 
XI.1975, F. Saraiva coll., 0155, 00210476, J.R.I. Ribeiro, 1998 det.; 1 male, pinned, Manaus municipality, campus INPA, 30.IV.1976, Paraluppi coll., 0020939, J.R.I. Ribeiro, 1998 det.; 1 female, pinned, Manaus municipality, campus INPA, 22.VI.1996, Paraluppi coll., 0020933, J.R.I. Ribeiro, 1998 det.; 1 male, pinned, Manaus municipality, campus INPA, 25.V.1986, J.R.I. Ribeiro, 1998 det.; 1 female, pinned, Manaus municipality, campus INPA, 16.VI.1976, E. Rufino coll., 0020929, J.R.I. Ribeiro det.; 12 males and 18 females, acohol 70\%, Manaus municipality, Centro de Projetos e Estudos Ambientais do Amazonas - Cepeam/Soka Gakkai, 0306'44.1"S 5954'22.7"W, 11.XII.2012, T.M. Almeida, G.P.S. Dantas, G.A. Gusmão, D. Colpani \& J.S. Oliveira colls., T.M. Almeida, 2013 det.; 2 females, ethyl alcohol 70\%, Manaus municipality, Centro Universitário do Norte-UNINORTE, 07.IX.2012, K. Sousa coll., T.M. Almeida, 2013 det.; 1 female, ethyl alcohol 70\%, Manaus municipality, Centro Universitário Uninorte, 07.IX.2012, T.M. Almeida coll., T.M. Almeida, 2013 det.; 1 female, pinned, Manaus municipality, Educandos district, 21.IX.1983, J.M. Rodrigues coll., 0021004, J.R.I. Ribeiro, 1998 det.; 2 males, pinned, Manaus municipality, AM 010, km 64, 1998, 29. VII.1970, A. Faustino coll., 3013, 0021044, J.R.I. Ribeiro det.; 1 male, pinned, Manaus municipality, Aleixo district, INPA, 06.XI.1975, F. Saraiva coll., 0155, 0021047, J.R.I. Ribeiro, 1998 det.; 1 female, pinned, Manaus municipality, INPA, 03.VII.1976, Mario Dantas coll., 1312, 0021003, J.R.I. Ribeiro, 1998 det.; 1 male, ethyl alcohol 70\%, Manaus municipality, Negro river, 03.X.2002, D.C.C. Pereira coll., A.L. de Melo det.; 1 male, ethyl alcohol 70\%, Manaus municipality, Negro river, 03.X.2002, D.L.V. Pereira det.; 1 female, ethyl alcohol 70\%, Manaus municipality, Puraquequara lake, $03^{\circ} 03^{\prime} 48.9^{\prime \prime S} 59^{\circ} 50^{\prime} 46.3^{\prime \prime} \mathrm{W}$, aquatic insect net, 13.XII.2012, T.M. Almeida, G.A. Gusmão, D. Colpani \& J.S. Oliveira colls. T.M. Almeida, 2013 det.; 1 female, ethyl alcohol 70\%, Manaus municipality, campus INPA, 19.V.2003,T.M. Almeida, 2013 det.; 1 male, pinned, Manaus municipality, 04-29.IV.2005, T.M. Almeida, 2013 det.; 1 male and 1 female ethyl alcohol 70\%, Manaus municipality, Coroado district, 25.VII.2012. T.M. Almeida coll., T.M. Almeida, 2013 det.; 2 males and 1 female, ethyl alcohol 70\%, Manaus municipality, 7 de Setembro avenue, downtown, 20.IX.2012. Silva, C.N. coll., T.M. Almeida, 2013 det.; 1 male, ethyl alcohol 70\%, Manaus municipality, INPA, campus II, in light, 25.II.2014, T. Mahlmann coll., T.M. Almeida, 2013 det.; 1 female, pinned, Manaus municipality, 13.III.1980, F. Peralta coll., 0020878, 0021006, J.R.I. Ribeiro, 1998 det.; 1 female, pinned, Manaus municipality, 26.VI.1976, M. Serrano coll., 2993, J.R.I. Ribeiro, 1998 det.; 1 female, pinned, Manaus municipality, Bosque da

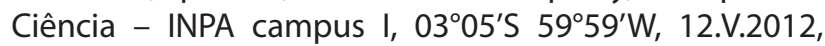
aquatic insect net, P.R. Bartholomay coll., T.M. Almeida, 2013 det.; 1 male, pinned, Manaus municipality, Puraquequara lake, 16-19.IX.2010, Ulysses coll., 0020924, T.M. Almeida, 2013 det.; 3 males, pinned, Manaus municipality, ZF-2 tower, $\mathrm{km} \mathrm{14,} 40$ metros de altura, $02^{\circ} 35^{\prime} 21^{\prime \prime} \mathrm{S}$ $60^{\circ} 06^{\prime} 55^{\prime \prime} \mathrm{W}$, mixed light and BLB, 16-19.VII.2004, J.A. Rafael, C.S. Motta, F.F. Xavier Fo, J.M.F. Ribeiro \& S. Trovisco colls., T.M. Almeida, 2013 det.; 1 male, pinned, Manaus municipality, Fucapi, Bloco E, 05.V.2010, P.Y. Oliveira coll.,
T.M. Almeida, 2018 det.; 1 male, pinned, Manaus municipality, Adrianópolis district, Belo Horizonte street, aquatic insect net, 07.V.2008, J.F. Barbosa coll., T.M. Almeida, 2018 det.; 1 male, pinned, Manaus municipality, Coroado district, INPA, 25.IV.2005, B.M. Oliveira coll., T.M. Almeida, 2018 det.; 1 female, pinned, Manaus municipality, INPA campus I, Aleixo district, 20.V.2011, D.T.A.S. Vidovix coll., T.M. Almeida, 2018 det.; 1 female, pinned, Manaus municipality, Adolpho Ducke reserve, AM 010, 0255'51"S 5958'59"W, 07.II.2008, G.L.S. Monte coll., T.M. Almeida, 2018 det.; 1 male, pinned, Manaus municipality, Ducke Reserve, 0255'49"S 5958'31"W, 07.V.2011, aquatic insect net, A.H.C. Oliveira coll., Belostomatidae A.H.C. Oliveira, 2011 det., T.M. Almeida, 2018 det.; 1 male and 1 female, ethyl alcohol 92\%, Manaus municipality, Bosque da Ciência - INPA campus I, 13.IV.2018, T.M. Almeida \& F. Stefanello colls., F. Stefanello, 2018 det.; 1 female, pinned, near Manaus municipality, AM 010 km 232, 01.IX.1977, L.P.A.E. Rufino coll., 0020916, J.R.I. Ribeiro, 1998 det.; 3 males, pinned, near Manaus municipality, tower ZF-2 Reserve, 45 km, 02³5'20"S 6006'55"W, 06-09.I.1997, C.S. Motta \& J. Vidal coll., 0016976, J.R.I. Ribeiro, 1998 det.; 1 male, pinned, near Manaus municipality, BR 174, km 2.5, F.G.L. Oliveira coll., Belostomatidae F.G.L. Oliveira det., T.M. Almeida, 2018 det.; 1 male, pinned, near Manaus municipality, AM 010, km 64, VIII.1970, A. Faustino coll., 0021042, 3106.27, J.R.I. Ribeiro, 1998 det.; 1 male, pinned, Manaus-Itacoatiara municipalities, Aruanã farm, AM 010, km 215, light trap, 22-23.VIII.1990, C.S. Motta et al. colls., 0021014, J.R.I. Ribeiro, 1998 det.; 1 female, pinned, Manacapuru municipality, Ressaca lake, 26.XII.1959, 0020894, 1966, H. Sioli \& W. Sattler, H.H. Weber det.; 1 male, pinned, Manicoré municipality, waterfall, $05^{\circ} 29^{\prime} 44^{\prime \prime S} 60^{\circ} 49^{\prime} 21^{\prime \prime} \mathrm{W}$, mixed light and BLB, IX.2004, Silva \& Pena colls., T.M. Almeida, 2013 det.; 2 males, pinned, Novo Aripuanã municipality, Xadá lake, 05'15'39"S 6042'32"W, mixed light and BLB, IV.2005, F. Xavier, F. Godoi \& A. Lourido colls., T.M. Almeida, 2013 det.; 1 male (with eggs) and 1 female, ethyl alcohol 70\%, Parintins municipality, Comprido's lake, near Amazonas river, 02²9'55.1"S 56²9'26.9"W, A19L, 25.IX.2003, P. de Marco \& N. Ferreira-Jr., V.P. Alecrim det.; 2 males, pinned, Presidente Figueiredo municipality, BR 174, km 113, 02²8'56"S 6001'48"W, mixed light trap, 07-08.VIII.1999, C.S. Motta et al. colls., T.M. Almeida, 2017 det.; 1 female, pinned, Presidente Figueiredo municipality, AM 240, km 12, Água Viva farm, mixed light trap, 18-22.XII.2006, F.F. Xavier Fo, G.M. Lourido, P.K.M. Almeida \& D.M.M. Mendes colls., T.M. Almeida, 2013 det.; 3 females, pinned, Presidente Figueiredo municipality, BR 174, km 200, light trap, 27.I.2006, J.A. Rafael; F.F. Xavier Fo, A. Silva \& D.M. Mendes colls., T.M. Almeida, 2013 det.; 2 females, pinned, Presidente Figueiredo municipality, BR 174, km 180, mobile light trap, 25-26.I.2006, J.A. Rafael, F.F. Xavier Fo, A. Silva, D.D. Mendes colls., T.M. Almeida, 2013 det.; 3 females, ethyl alcohol 70\%, Presidente Figueiredo municipality, BR 174, km 21, 0247'31.8"S 06002'04.6"W, 10. XII.2012, G.A. Gusmão, D. Colpani, T.M. Almeida, G.P.S. Dantas \& J.S. Oliveira colls., T.M. Almeida, 2013 det.; 1 male, pinned, Rio Preto da Eva municipality, S. Petiza 
coll., IV.2009, Belostomatidae E.L.L. Belmont det., T.M. Almeida, 2018 det.; 1 male, pinned, São Paulo de Olivença municipality, 18.IX.1961, E. Faustino coll., 2292, 0021050, J.R.I. Ribeiro, 1998 det.; 1 male, ethyl alcohol 70\%, Tabatinga municipality, $04^{\circ} 12^{\prime} 15^{\prime \prime} \mathrm{S} 69^{\circ} 54^{\prime} 32^{\prime \prime} \mathrm{W}$, light trap, 08.IX.2005, J.A. Rafael \& F.F. Xavier Fo colls., T.M. Almeida, 2013 det.; 1 male, pinned, Tefé municipality, EMADE high-

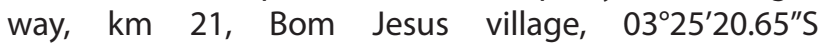
64³7'05.70"W, mixed light trap, 29-30.VII.2016, D.M.M. Mendes \& J.C. Oliveira colls., T.M. Almeida, 2018 det.; 1 female, pinned, near Tefé municipality, Tefé lake, near Solimões river, 10.XII.1976, E. Faustino coll., 0020927, J.R.I. Ribeiro, 1998 det.; 1 male and 1 female, ethyl alcohol 70\%, Comprido's lake, near Amazonas river, 02²9'55.1"S 56²9'26.9"W, A19L, 25.IX.2003, P. de Marco \& N. Ferreira-Jr., V.P. Alecrim det. 2 males (1 with eggs), ethyl alcohol 70\%, Pará, Alenquer municipality, Bom lugar river, 06-08.VII.1985, A. Celeste coll., T.M. Almeida, 2013 det.; 1 male and 1 female, ethyl alcohol 70\%, Almerim municipality, Paranaquara lake, near Amazonas river,

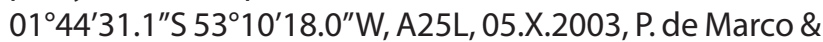
N. Ferreira-Jr., V.P. Alecrim det.; 1 male, pinned, Bragantina municipality, Quatipuru, 05.IV.1963, E.J. Fittkau coll., 0020897, H.H. Weber, 1966 det.; 1 female, ethyl alcohol 70\%, Óbidos municipality, Januária, Ipapucu lake, near

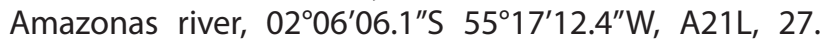
IX.2003, P. de Marco \& N. Ferreira-Jr., V.P. Alecrim det.; 2 females, ethyl alcohol 70\%, Óbidos, Curuçambá stream, $01^{\circ} 50^{\prime} 04^{\prime \prime} \mathrm{S} 55^{\circ} 29^{\prime} 26^{\prime \prime} \mathrm{W}$, light trap, 10.IX.2001, J.A. Rafael \& J.F. Vidal colls., T.M. Almeida, 2013 det.; 1 female, ethyl alcohol 70\%, Santarém municipality, Santana do Ituqui, Maicá lake, 02³0'44.0"S 54¹9'43.9”W, A22L, 30.IX.2003, P. de Marco \& N. Ferreira-Jr. colls., V.P. Alecrim det.; 1 female, ethyl alcohol 70\%, Santarém municipality, Santana do Ituqui, Maicá lake, near Amazonas river, 02³0'44.0"S 54¹9'43.9"W, A22L, 30.IX.2003, P. de Marco \& N. Ferreira-Jr. colls., V.P. Alecrim det. 1 male, ethyl alcohol 70\%, Rio Grande do Sul, Canela municipality, ARR, 21.IV.1989, U. Barbosa coll., T.M. Almeida, 2013 det. 3 males and 2 females, ethyl alcohol 70\%, Tocantins, Palmas municipality, Brejo da Jéssica stream, 1003'53.60"S 48¹4'58.00"W, 06.Vl.2016, A. Pes, G. Amora, J. Silva, N. Hamada, S. Couceiro \& J.P. Benevides colls., F. Stefanello, 2018 det.

Notes: This is the first record of B. discretum to Rio Grande do Sul state (Brazil).

\section{Oxyurum group sensu Ribeiro \& Estévez (2009)}

Diagnose: Small species; flat and broad ventral diverticulum; pilosity of the connexivum well developed.

\section{Belostoma candidulum Montandon, 1903}

(Figs. 2D, 7B)

Known distribution: ARGENTINA: Buenos Aires (Ribeiro, 2007). BRAZIL: Minas Gerais (Schnack, 1976), Goiás (Ribeiro, 2005), Rio de Janeiro (Ribeiro, 2005), Rio
Grande do Sul (Montandon, 1903a), São Paulo (Schnack, 1976), Santa Catarina (Ribeiro \& Estévez, 2009).

Material examined: 1 male and 1 female, ethyl alcohol 70\%, BRAZIL, Mato Grosso do Sul, Bodoquena municipality, Califórnia and Ouro Verde farms, waterfall, 2042'18.1"S 5651'14.6"W, 18.III.2012, N. Hamada, P. Vilela \& N. Zampiva colls., F. Stefanello, 2018 det.

Notes: This is the first record of $B$. candidulum to Mato Grosso do Sul state (Brazil).

\section{Belostoma carajaensis Ribeiro \& Estévez, 2009}

(Figs. 2E, 7B)

Known distribution: BRAZIL: Pará (Ribeiro \& Estévez, 2009).

Material examined: 1 male, ethyl alcohol 70\%, BRAZIL, Amazonas, Iranduba municipality, Manoel Urbano

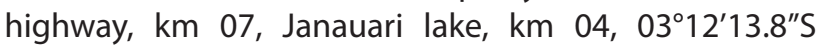
$60^{\circ} 08^{\prime} 02.2^{\prime \prime}$ W, 12.XII.2012, T.M. Almeida, G.A. Gusmão, D. Colpani, G.P.S. Dantas \& J.S. Oliveira colls., F. Stefanello, 2018 det.

Notes: This is the first record of $B$. carajaensis from Amazonas state (Brazil).

\section{Plebejum group sensu Nieser (1975)}

Diagnose: Small species; pilosity covering half or less of ventral laterotergites, ventro-apical protuberance in the diverticulum.

\section{Belostoma estevezae Ribeiro \& Alecrim, 2008}

(Figs. 2F, 8A)

Known distribution: BRAZIL: Mato Grosso. Tapirapé river (Ribeiro \& Alecrim, 2008; Ribeiro et al., 2018).

Material examined: 1 female, pinned, BRAZIL, Amazonas, Iranduba municipality, Marchantaria island, 26.II.1976, I.S. Gorayeb coll., 0020876, J.R.I. Ribeiro, 2006 det.

Notes: This is the first record of B. estevezae to Amazonas state (Brazil).

\section{Belostoma micantulum (Stål, 1860)}

(Figs. 2G, 8A)

Known distribution: ARGENTINA: Corrientes, Formosa, Salta (Ribeiro, 2007), Buenos Aires, Chaco, Entre Ríos, Misiones, Santiago del Estero, Itatí, Misiones, Santa Fe (De Carlo, 1930), Tucumán (Estévez, 1996), Rosario (Schnack, 1976), La Plata (Mayr, 1871). BOLIVIA: Chapare, Beni, 
Santa Cruz (Estévez \& Polhemus, 2007). BRAZIL: Amapá, Amazonas, Pará (Nieser, 1975), Ceará, Mato Grosso, Mato Grosso do Sul (Ribeiro, 2007), Goiás (Menke \& Lauck, 1962), Minas Gerais (Nieser \& Melo, 1997), Rio de Janeiro (Stål, 1860; Mayr, 1871), Rio Grande do Sul, Tocantins (Estévez \& Polhemus, 2007) (Ribeiro et al., 2018). COLOMBIA: Amazonas, Cuatro Bocas (Estévez \& Polhemus, 2007), Meta (Roback \& Nieser, 1974). GUYANA: Alexander Village, Georgetown, Karanambo, West Bank Demerata (Nieser, 1975). PARAGUAY: Alto Verá, Colonia Fernheim, Emboscada, Primavera, Sapucay, Trinidad (Estévez, 1996), Puerto Guaraní (De Carlo, 1938), Villarica (Ribeiro,

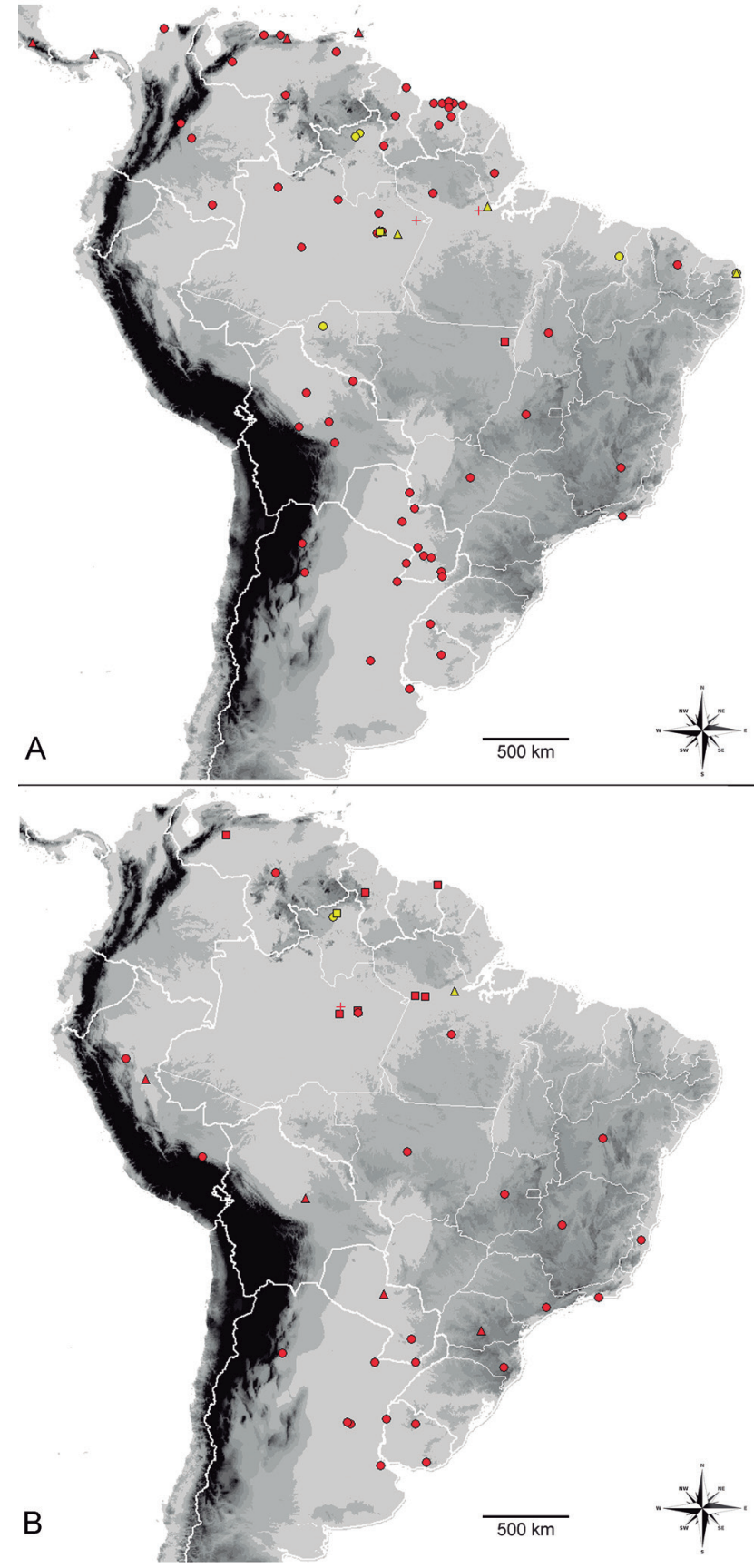

Figure 8. Distribution of Belostomatidae in South America. Red symbol: known records, yellow symbol: new records. $(A)=$ Belostoma estevezae (square), B. micantulum (circle), B. minusculum (triangle) [not all distributional records are shown on the map], $B$. nessimiani (cross). $(B)=B$. nicaem (cross), B. parvum (square), B. plebejum (circle), B. pygmeum (triangle).
2007). SURINAM: Brokopondo, Commewijne, Coronie, Marowijne, Saramacca, Paramaribo, Republiek, Zanderij (Nieser, 1975). URUGUAY: Artigas, Arroyo de la Invernada (Ruffinelli \& Pirán, 1959). VENEZUELA: Barinas, Caracas, Monagas, San Esteban (Estévez \& Polhemus, 2007).

Material examined: 4 males and 2 females, ethyl alcohol 70\%, BRAZIL, Amazonas, Barcelos municipality, in stream, 29.VIII.2009, PT1004, T.M. Almeida, 2018 det.; 1 female, ethyl alcohol 70\%, Careiro da Várzea municipality, Purupuru lake, km 35, BR 319, 08.VIII.2012, N. Hamada, J.O. Silva, N. Zampiva \& N. Hidalgo colls., T.M. Almeida, 2018 det.; 1 female, ethyl alcohol 70\%, Iranduba municipality, Manoel Urbano highway, km 7, Janauarí lake, km 8.5, 0313'47.4"S 6006'14.7"W, 12.XII.2012, T.M. Almeida, G.A. Gusmão, D. Colpani, G.P.S. Dantas \& J.S. Oliveira colls., T.M. Almeida, 2018 det.; 1 male, ethyl alcohol 70\%, Iranduba municipality, Bodó lake, Marchantaria island, 02.X.2002, D.L.V. Pereira coll., T.M. Almeida, 2018 det.; 1 female, pinned, Iranduba municipality, 28.XII.1985, F.A. Peralta coll., 0021026, J.R.I. Ribeiro, 2004 det.; 1 female, ethyl alcohol 70\%, Manaus municipality, Camaleão lake, Marchantaria island, 30.IX.2002, D.L.V. Pereira coll., A.L. Melo det.; 1 male, ethyl alcohol 70\%, Presidente Figueiredo municipality, BR 174, km 124, Santa Cruz stream, $01^{\circ} 54^{\prime} 09.0^{\prime \prime S} \quad 60^{\circ} 03^{\prime} 48.3^{\prime \prime} \mathrm{W}$, 10.XII.2012, T.M. Almeida, G.A. Gusmão, D. Colpani, G.P.S. Dantas \& J.S. Oliveira colls., T.M. Almeida, 2018 det.; 1 female, ethyl alcohol 70\%, São Gabriel da Cachoeira municipality, Morro dos Seis Lagos, Vulcão lake, 09.X.1990, team Neblina colls., T.M. Almeida, 2018 det.; 1 male, ethyl alcohol 70\%, São Gabriel da Cachoeira municipality, Morro dos Seis Lagos, Vulcão lake, 09.X.1990, team Neblina colls., T.M. Almeida, 2018 det.; 1 female, ethyl alcohol 70\%, São Gabriel da Cachoeira municipality, Dragão lake, 07.X.1990, team Neblina colls., T.M. Almeida, 2018 det.; 1 female, ethyl alcohol 70\%, Taboca stream, 134, 08.VIII.1985, team Gavião colls., T.M. Almeida, 2018 det.; 2 males and 5 females, ethyl alcohol 70\%, Manilulaú stream, 08.VIII.2009, PT884, T.M. Almeida, 2018 det.; 1 female, pinned, Maranhão, Caxias municipality, 04 $53^{\prime} 26.0^{\prime \prime} \mathrm{S} 43^{\circ} 24^{\prime} 38.2^{\prime \prime} \mathrm{W}$, light trap, 17.V.2007, J.A. Rafael, F.F. Xavier Fo \& F.L. Oliveira colls., T.M. Almeida, 2013 det. 2 females, ethyl alcohol 70\%, Mato Grosso, Vila Bela da Santíssima Trindade municipality, Guaporé river, 01.X.1984, team Rosé colls., 23, 1811, T.M. Almeida, 2018 det. 1 male and 1 female, ethyl alcohol 70\%, Pará, Porteira waterfall, Trombetas river, 10.IV.1985, team Pyriquito colls., T.M. Almeida, 2018 det.; 1 female, ethyl alcohol 70\%, Trombetas river, Juquiri, 08.III.1986, team Apoidea colls., 1745, T.M. Almeida, 2018 det.; 2 males, ethyl alcohol 70\%, Anã waterfall, 13.X.1985, team Comander colls., M.E. Lanzer-de-Souza, 1987 det.; 3 males, ethyl alcohol 70\%, Tocantins river, 12.VII.1987, Ulysses Barbosa coll., T.M. Almeida, 2018 det.; 1 female, ethyl alcohol 70\%, PA 150, Sabiá river, 13.IV.1988, Ulysses Barbosa coll., 1712, T.M. Almeida, 2018 det.; 1 female, ethyl alcohol 70\%, Boa Sorte farm, Boa Sorte stream, 22.IV.1988, Ulysses Barbosa coll., 181, T.M. Almeida, 2018 det. 1 male, ethyl alcohol 70\%, Rio Grande do Norte, Mipibu river, IX.1991, Andrade coll., T.M. Almeida, 2018 det. 1 female, ethyl alcohol 70\%, 
Rondônia, Bom Futuro stream, BR 421, 30.VII.1985, team Gavião, T.M. Almeida, 2018 det.; 1 male, ethyl alcohol 70\%, Mutum river, 14:06, 10.VIII.1985, team Gavião, T.M. Almeida, 2018 det.; 1 female, ethyl alcohol 70\%, in stream, 11:34, 16.VIII.1985, team Gavião colls, T.M. Almeida, 2018 det.; 1 male, ethyl alcohol 70\%, river, 14.VIII.1985, team Gavião colls., T.M. Almeida, 2018 det.; 1 male, ethyl alcohol 70\%, in stream, 10:40, 10.VIII.1985, team Gavião, T.M. Almeida, 2018 det. 1 female, ethyl alcohol 70\%, Roraima, Amajari municipality, Mururi stream, near Uraricoera river, 23.X.1987, team Grandfinale colls., T.M. Almeida, 2018 det.; 1 female, ethyl alcohol 70\%, Amajari municipality, near Uraricoera river, Maracá Ecological Station, light trap, 25.IX.2011, T.M. Almeida, 2018 det.; 1 female, ethyl alcohol 70\%, Pedra Sentada stream, 05.IV.1987, team Ericó colls., T.M. Almeida, 2018 det.

Notes: This is the first record of $B$. micantulum from Maranhão, Rio Grande do Norte, Rondônia and Roraima states (Brazil).

\section{Belostoma minusculum (Uhler, 1884)}

(Figs. 2H, 8A)

Known distribution: COSTA RICA: San José, Puntarenas (Estévez \& Polhemus, 2007). HONDURAS: Choluteca (Estévez \& Polhemus, 2007). NICARAGUA: Bonanza, La Trinidad, Somoto (Uhler, 1884; Estévez \& Polhemus, 2007). PANAMA: Barro Colorado Island (Estévez \& Polhemus, 2007). TRINIDAD AND TOBAGO: Barataria (Estévez \& Polhemus, 2007). VENEZUELA: Barinitas, Guárico, Miranda (Estévez \& Polhemus, 2007).

Material examined: 1 female, ethyl alcohol 70\%, BRAZIL, Amazonas, Ataiana stream, near Negro river, 10.VIII.2009, PT1063, T.M. Almeida, 2018 det.; 1 male, ethyl alcohol 70\%, Careiro da Várzea municipality, 08.IX.2011, H.D.D. Rodrigues coll., T.M. Almeida, 2018 det.; 2 females, ethyl alcohol 70\%, Itacoatiara municipality, Amazonas river, Trindade island, $03^{\circ} 18^{\prime} 48.4^{\prime \prime S} 58^{\circ} 43^{\prime} 56.3^{\prime \prime} \mathrm{W}$, UV light trap, A17UV, 22.IX.2003, P. de Marco \& N. Ferreira-Jr. colls., V.P. Alecrim det.; 1 male, pinned, Manaus municipality, km 43, 25.III.1982, J.W. Morais coll., 16, 0021025, J.R.I. Ribeiro, 2004 det. 1 male, ethyl alcohol 70\%, Pará, Almeirim municipality, Amazonas river, Novo Aramanduba, 01²7'11.9"S 52²9'48.4"W, A26L, 07.X.2003, P. de Marco \& N. Ferreira-Jr. colls., V.P. Alecrim det. 4 males and 7 females, ethyl alcohol 70\%, Rio Grande do Norte, São José do Mipibu municipality, VIII.1991, Hebert coll., T.M. Almeida, 2018 det.

Notes: This is the first record of B. minusculum from Brazil (Amazonas, Pará and Rio Grande do Norte states).

\section{Belostoma nessimiani Ribeiro \& Alecrim, 2008} (Figs. 2I, 8A)

Known distribution: BRAZIL: Amazonas, Pará (Ribeiro \& Alecrim, 2008; Ribeiro et al., 2018).
Type Material examined: 1 male holotype, ethyl alcohol 70\%, BRAZIL, Amazonas, Albano lake, near Amazonas river, 02²4'51.1"S 57²9'59.8"W, 24.IX.2003, UV light trap, P. de Marco \& N. Ferreira-Jr. colls., J.R.I. Ribeiro \& V.P. Alecrim, 2006 det.; 1 male and 1 female paratypes, ethyl alcohol 70\%, Albano lake, near Amazonas river, $02^{\circ} 24^{\prime} 51.1^{\prime \prime} \mathrm{S} 57^{\circ} 29^{\prime} 59.8^{\prime \prime} \mathrm{W}$, UV light trap, 24.IX.2003, P. de Marco \& N. Ferreira-Jr. colls., J.R.I. Ribeiro \& V.P. Alecrim, 2006 det.; 1 male paratype, ethyl alcohol 70\%, Pará, Almerim municipality, Paranaquara lake, UV light trap, $01^{\circ} 44^{\prime} 31.1^{\prime \prime} \mathrm{S} 53^{\circ} 10^{\prime} 18.0^{\prime \prime} \mathrm{W}, 05 . X .2003$, P. de Marco \& N. Ferreira-Jr. colls., J.R.I. Ribeiro \& V.P. Alecrim, 2006 det.

Additional Material examined: 1 male, ethyl alcohol 70\%, BRAZIL, Amazonas, Barcelos municipality, 25.IX.2014, A.M.O. Pes, G.D. Gomes \& D. Colpani, colls., F. Stefanello, 2018 det.

\section{Belostoma nicaem Estevez \& J. Polhemus, 2007} (Figs. 2J, 8B)

Known distribution: BRAZIL: Amazonas (Estévez \& Polhemus, 2007; Ribeiro et al., 2018).

Material examined: 1 female, pinned, BRAZIL, Amazonas, Manaus municipality, V.1962, several collectors, 2419, 0021056, J.R.I. Ribeiro, 2004 det.

\section{Belostoma parvum Estévez \& Polhemus, 2007} (Figs. 2K, 8B)

Known distribution: BRAZIL: Pará (Estévez \& Polhemus, 2007). COLOMBIA: Amazonas (Estévez \& Polhemus, 2007). GUYANA: Supuruni (Estévez \& Polhemus, 2007). SURINAM: Moengo (Estévez \& Polhemus, 2007). VENEZUELA: Portuguesa (Estévez \& Polhemus, 2007; Ribeiro et al., 2018).

Material examined: 1 female, ethyl alcohol 70\%, BRAZIL, Amazonas, Amazonas river, Caapiranga municipality, Prainha, Mureru lake, A23L, 02.X.2003, P. de Marco \& N. Ferreira-Jr. colls., V.P. Alecrim det.; 3 males and 3 females, ethyl alcohol 92\%, Manaus municipality, Bosque da Ciência - INPA campus I, 13.IV.2018, T.M. Almeida \& F. Stefanello colls., F. Stefanello, 2018 det. 1 male, ethyl alcohol 70\%, Roraima, Amajari municipality, Uraricoera river, Ericó village, in stream, 31.VIII.1987, team Ericó colls., T.M. Almeida, 2018 det. 1 female, ethyl alcohol 70\%, Pará, Óbidos municipality, Januária, Ipapucu lake, near Amazonas river, 0206'06.1"S 55¹7'12.4"W, A21L, 27.IX.2003, P. de Marco \& N. Ferreira-Jr. colls., V.P. Alecrim det.; 1 female, ethyl alcohol 70\%, Juruti municipality, Recreio, Recreio lake, near Amazonas river, 0203'57.9"S 5557'33.7"W, A20L, 26.IX.2003, P. de Marco \& N. Ferreira-Jr. colls., V.P. Alecrim det.; 1 male, ethyl alcohol 70\%, Caetano lake, near Trombetas river, 09.III.1986, team Apoidea colls., 1741, T.M. Almeida, 2018 det.; 1 female, ethyl alcohol 70\%, 
BR 230, Jaú river, 23.IV.1988, Ulysses Barbosa coll., 1709, T.M. Almeida, 2018 det.

Notes: This is the first record of B. parvum from Amazonas and Roraima states (Brazil).

\section{Belostoma plebejum (Stål, 1860)}

(Figs. 2L, 8B)

Known distribution: ARGENTINA: Buenos Aires, Concordia, Corrientes, Entre Ríos, Misiones, Santa Fe, Tucumán (Estévez \& Polhemus, 2007; Ribeiro et al., 2018). BRAZIL: Amazonas, Bahia, Espírito Santo, Goiás, Mato Grosso, Minas Gerais, Pará, Rio de Janeiro, São Paulo, Santa Catarina (Stål, 1860; Estévez \& Polhemus, 2007; Ribeiro et al., 2018). PARAGUAY: Alvobena, Guairá (Estévez \& Polhemus, 2007; Ribeiro et al., 2018). PERU: Marañón River, Quincemil (Estévez \& Polhemus, 2007; Ribeiro et al., 2018). URUGUAY: Lavalleja, Tacuarembó (Estévez \& Polhemus, 2007; Ribeiro et al., 2018). VENEZUELA: (Estévez \& Polhemus, 2007; Ribeiro et al., 2018).

Material examined: 1 male, ethyl alcohol 70\%, BRAZIL, Amazonas, Iranduba municipality, Camaleão lake, Marchantaria island, 30.IX.2002, D.L.V. Pereira coll., A.L. Melo det.; 1 female, ethyl alcohol $70 \%$, Iranduba municipality, Redondo lake, Marchantaria island, 30.IX.2002, D.L.V. Pereira coll., A.L. Melo det.; 1 female, ethyl alcohol $70 \%$, Presidente Figueiredo municipality, Onça stream, AM 240, km 20, 11.IX.2002, D.L.V. Pereira coll., A.L. Melo det. 1 male, ethyl alcohol 70\%, Roraima, Amajari municipality, Maracá Ecological Station, Pedra Sentada stream, near Uraricoera river, 05.IX.1987, team Ericó coll., T.M. Almeida, 2013 det.

Notes: This is the first record of B. plebejum from Roraima state (Brazil).

\section{Belostoma pygmeum Dufour, 1863 (Figs. 3A, 8B)}

Known distribution: BOLIVIA: unknow Department (Estévez \& Polhemus, 2007). BRAZIL: Amazonas, Paraná (Estévez \& Polhemus, 2007; Ribeiro et al., 2018). PARAGUAY: unknow Departament: (Estévez \& Polhemus, 2007). PERU: unknow Region (Estévez \& Polhemus, 2007).

Material examined: 1 male, ethyl alcohol 70\%, BRAZIL, Amazonas, Manaus municipality, campus F.U.A, light trap, ESALQ, 27-28.IX.1982, T.M. Almeida, 2018 det.; 1 male, pinned, Manaus municipality, 05.XI.1962, several collectors, 0021057, 2478, J.R.I. Ribeiro, 2004 det.; 4 males and 5 females, ethyl alcohol 92\%, Manaus municipality, Bosque da Ciência - INPA campus I, 13.IV.2018, T.M. Almeida \& F. Stefanello colls., F. Stefanello, 2018 det. 1 male, ethyl alcohol 70\%, Pará, Almerim municipality, Paranaquara lake, near Amazonas river, A25L, 05.X.2003, P. de Marco \& N. Ferreira-Jr. colls., V.P. Alecrim det.
Notes: This is the first record of $B$. pygmeum from Pará state (Brazil).

\section{Testaceopallidum group sensu Lauck (1963)}

Diagnose: Pilosity covering entirely the connexivum and part of the sternites; rostrum with first segment distinctly shorter than the second.

\section{Belostoma ribeiroi De Carlo, 1933 (Figs. 3B, 9A)}

Known distribution: BRAZIL: Distrito Federal, Rio de Janeiro, Santa Catarina (Ribeiro, 2007), Goiás (Menke \& Lauck, 1962), Mato Grosso (De Carlo, 1933), Minas Gerais (Nieser \& Melo, 1997).

Material examined: 2 females, ethyl alcohol $70 \%$, BRAZIL, Mato Grosso do Sul, Bodoquena municipality, Califórnia and Ouro Verde farms, $20^{\circ} 42^{\prime} 18.1^{\prime \prime} \mathrm{S}$ $56^{\circ} 51^{\prime} 14.6^{\prime \prime} \mathrm{W}$, 18.III.2012, N. Hamada, P. Vilela \& N. Zampiva colls., F. Stefanello, 2018 det. 1 female, ethyl alcohol 70\%, Mato Grosso, Chapada dos Guimarães, rio Jamacá (\#30) 15²7'47.4"S 5542'49.8"W, 19.VI.2014, N. Hamada \& J.M.C. Nascimento colls., F. Stefanello, 2018 det.

Notes: This is the first record of $B$. ribeiroi from Mato Grosso do Sul state (Brazil).

\section{Stollii group sensu Lauck (1963)}

Diagnose: Vertex with prominet carina; pilosity not covering entirely the connexivum.

\section{Belostoma stollii (Amyot \& Serville, 1843)} (Figs. 3C, 9B)

Known distribution: BOLIVIA: Santa Cruz (Ribeiro, 2000). BRAZIL: Amazonas (Lauck, 1963), Mato Grosso (Lauck, 1963), Minas Gerais (Nieser \& Melo, 1997), Pará, Rio de Janeiro (Ribeiro, 2000), unknow State (De Carlo, 1938). FRENCH GUYANA: Cayenne (Amyot \& Serville, 1843; Ribeiro, 2007), Pariacabo (Lauck, 1963). GUYANA: (De Carlo, 1938). Supuruni Creek (Nieser, 1975). PERU: (Ribeiro, 2005). Huanuco. SURINAM: Brokopondo, Marowijne, Nickerie (Nieser, 1975), Saracreek (Ribeiro, 2007), unknow District (Lauck, 1963). VENEZUELA: Amazonas (Lauck, 1963).

Material examined: 1 male, pinned, BRAZIL, Amazonas, Barcelos municipality, Coruja stream, near Ererê river, $00^{\circ} 06^{\prime} 16^{\prime \prime} \mathrm{N} 63^{\circ} 51^{\prime} 01^{\prime \prime} \mathrm{W}$, mixed light trap, 12-25.VIII.2009, F.F. Xavier Fo, G.L.S. Monte \& R. Alencar colls. T.M. Almeida, 2018 det.; 3 males, pinned, Borba municipality, Abacaxis river, Paxiúba, 04²8'48"S 
58 34'24"W, light trap, 02-04.VI.2008. J.A. Rafael et al. coll., T.M. Almeida, 2018 det.; 3 males, pinned, Manaus municipality, ZF-2 Reserve, km 14, 02 $35^{\circ} 21^{\prime \prime} \mathrm{S}$ $60^{\circ} 06^{\prime} 55^{\prime \prime} \mathrm{W}$, mixed light and BLB, 16-19.VII.2004, J.A. Rafael, C.S. Motta, F.F. Xavier Fo, J.M.F. Ribeiro \& S. Trovisco colls., T.M. Almeida, 2018 det.; 1 female, pinned, near Manaus municipality, ZF-2 Reserve, km 14, 40 m, $02^{\circ} 35^{\prime} 21^{\prime \prime} \mathrm{S} 60^{\circ} 06^{\prime} 55^{\prime \prime} \mathrm{W}$, mixed light and BLB, 18-21. II.2004, J.A. Rafael, C.S. Motta, F.F. Xavier Fo, J.M.F. Ribeiro \& S. Trovisco colls., T.M. Almeida, 2018 det.; 1 male, pinned, near Manaus municipality, ZF-2 Reserve, mixed light trap, 04-06.VII.2013, F.F. Xavier Fo, A. Agudelo, C. Maldaner \& D.M. Mendes colls., Belostomatidae D. Mendes det., T.M. Almeida, 2018 det.; 2 females, pinned,
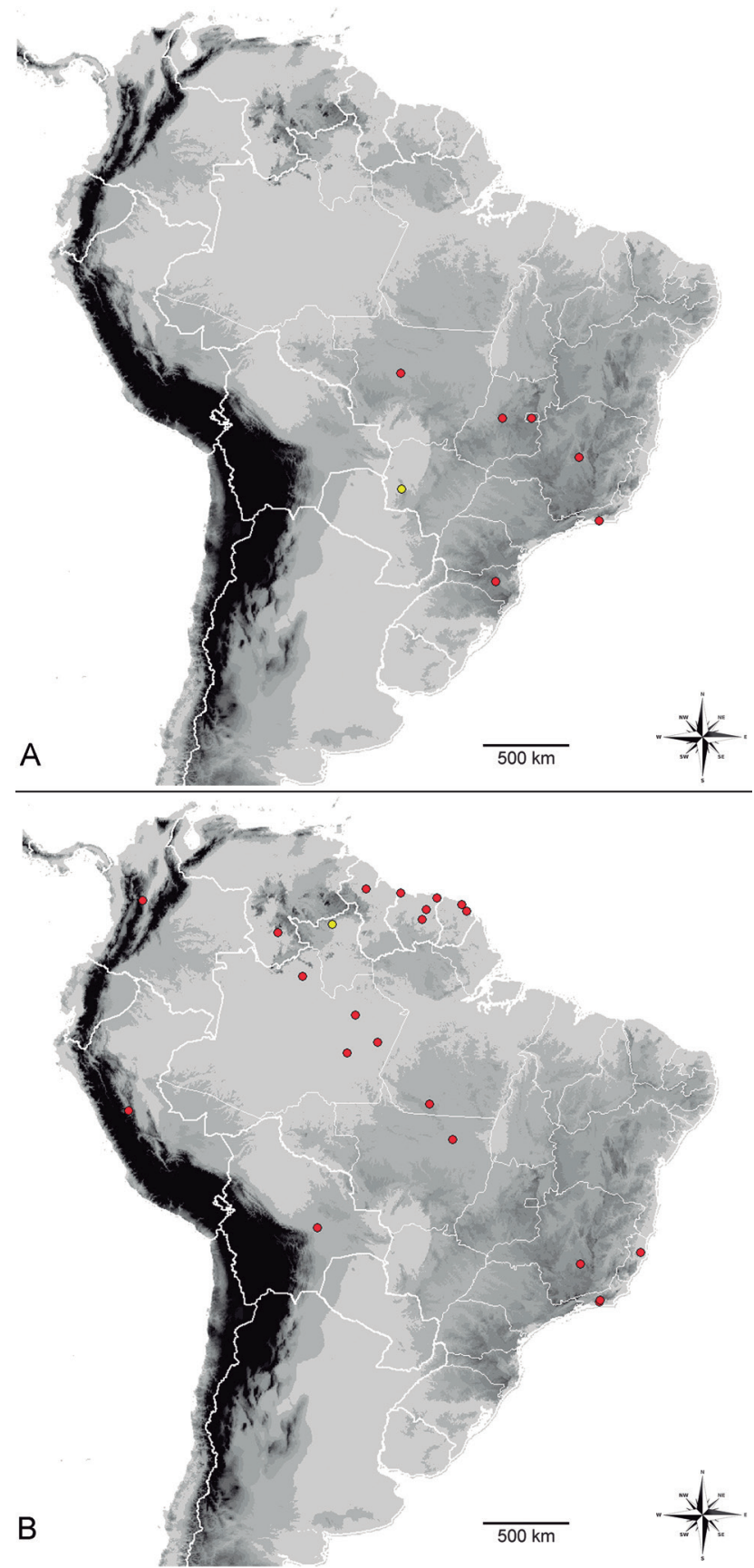

Figure 9. Distribution of Belostomatidae in South America. Red symbol: known records, yellow symbol: new records. $(A)=$ Belostoma ribeiroi. $(B)=$ B. stollii.
Novo Aripuanã municipality, $05^{\circ} 15^{\prime} 39^{\prime \prime} \mathrm{S} 60^{\circ} 42^{\prime} 32^{\prime \prime} \mathrm{W}$, mixed light and BLB, IV.2005, F.F. Xavier Fo, F. Godoi \& A. Lourido colls., T.M. Almeida, 2018 det.; 1 male, pinned, Presidente Figueiredo municipality, AM 240, km 24, 02³5'21"S 6006'55"W, 11-12.XII.2004, F.F. Xavier Fo \& G.M. Lourido colls., T.M. Almeida, 2013 det. 1 male, pinned, Roraima, Amajari municipality, Serra

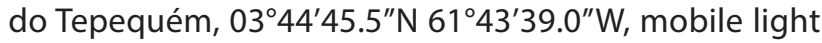
trap, 10-11.VII.2010, J.A. Rafael \& T. Krolow colls., T.M. Almeida, 2018 det.

Notes: This is the first record of $B$. stollii to Roraima state (Brazil).

\section{Triangulum group sensu Lauck, 1964}

Diagnose: Prosternal keel triangular; pilosity covering one-third of connexivum.

\section{Belostoma bachmanni De Carlo, 1956 (Figs. 3D, 10A)}

Known distribution: Known only from the locality of the type series (Paraguay, Department. Concepción, Puerto Vallemí) (De Carlo, 1956) and from Brazil, Purus river and Solimões river (Estévez, unpublished data).

Material examined: 3 females, ethyl alcohol 70\%, BRAZIL, Amazonas, Novo Airão municipality, Anavilhanas National Park, 01.IX.2001, G. Machado coll., F. Stefanello, 2018 det.; 1 male and 2 females, ethyl alcohol 70\%, São Gabriel da Cachoeira municipality, 07.VIII.2010, R.L. Ferreira-Keppler \& S.R.S. Torreias coll., F. Stefanello, 2018 det.; 1 male and 2 females, ethyl alcohol 70\%, Barcelos municipality, Aracá river, $00^{\circ} 24^{\prime} 13.8^{\prime \prime} \mathrm{S} 62^{\circ} 55^{\prime} 57.6^{\prime \prime} \mathrm{W}$, 25.IX.2014, A.M.O. Pes et al. colls., F. Stefanello, 2018 det.; 1 male, ethyl alcohol 70\%, Santa Isabel do Rio Negro municipality, Jaradi stream, $00^{\circ} 20^{\prime} 14.2^{\prime \prime S} 65^{\circ} 12^{\prime} 25.7^{\prime \prime} \mathrm{W}, 18 . I X .2014$. A.M.O. Pes et al. colls., F. Stefanello, 2018 det.; 4 males and 6 females, ethyl alcohol 70\%, Maruiá river, 19.IX.2014. A.M.O. Pes et al. colls., F. Stefanello, 2018 det.

Notes: This species is poorly cited in the literature and unknown by many researchers studying giant water bugs. There were doubts about which species group Belostoma bachmanni belongs to and its range of distribution. Recently, Estévez (unpublished data) included this species in the B. triangulum group of Lauck (1964), considering the triangular prosternal keel and male genitalia, which have arms of phalobase long, extending beyond the flat ventral diverticulum. The males examined were 19.5-20.0 mm in length and the greatest body width were 9.6-10.2 $\mathrm{mm}$; females were 19.8-21.0 $\mathrm{mm}$ in length and the greatest body width were 9.5-10.2 mm, both male and female have flattened body. This species appears to be relatively abundant along the Negro river and its tributaries. 


\section{Belostoma fittkaui De Carlo, 1966} (Figs. 3E, 10A)

Known distribution: Known only from the type series, which only includes males (Cachoeira stream, Cuieiras river, Amazonas, Brazil) (De Carlo, 1966).

Material examined: 2 males and 2 females, ethyl alcohol 70\%, BRAZIL, Amazonas, Barcelos municipality, Demini river, $00^{\circ} 38^{\prime} 05.3^{\prime \prime}$ S 62 $51^{\prime} 52.7^{\prime \prime}$ W, 24.IX.2014, A.M.O. Pes et al. colls., F. Stefanello, 2018 det.; 1 female, ethyl alcohol $70 \%$, Santa Isabel do Rio Negro municipality, Maruiá river, 19.IX.2014, A.M.O. Pes et al. colls., F. Stefanello, 2018 det.
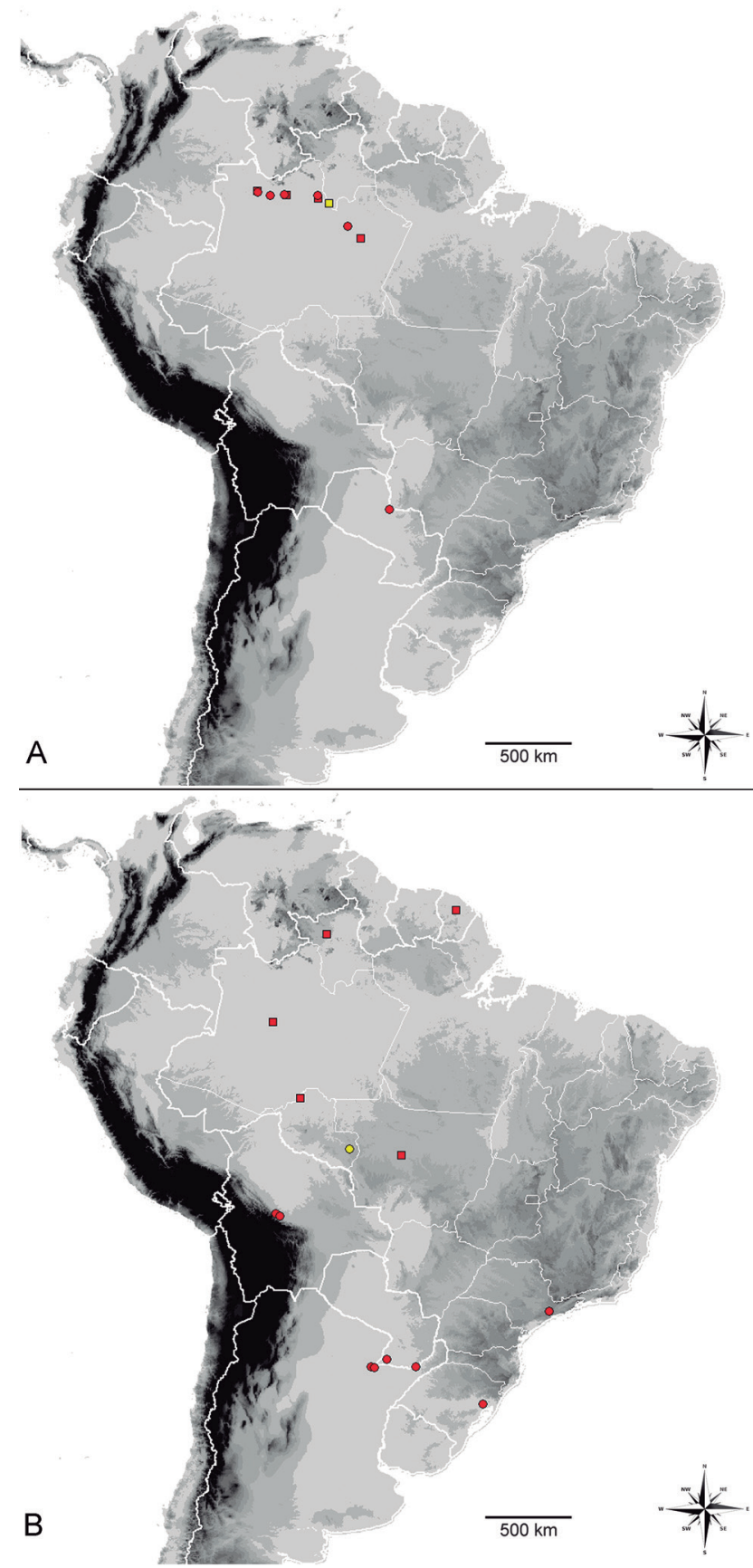

Figure 10. Distribution of Belostomatidae in South America. Red symbol: known records, yellow symbol: new records. (A) = Belostoma fittkaui (square), B. bachmanni (circle). (B) = Weberiella rhomboides (square), Horvathinia pelocoroides (circle).
1 female, ethyl alcohol 70\%, Careiro da Várzea municipality, Purupuru lake, BR 319, 08.VIII.2012, N. Hamada col., F. Stefanello, 2018 det. 3 males and 4 females, ethyl alcohol $70 \%$, Roraima, Caracaraí municipality, Pretinho stream, near Jufari river, $00^{\circ} 57^{\prime} 45.6^{\prime \prime} \mathrm{S} 62^{\circ} 06^{\prime} 40.2^{\prime \prime} \mathrm{W}$, 29.IX.2014, A.M.O. Pes et al. colls., F. Stefanello, 2018 det.

Notes: This species is poorly cited in the literature and unknown by many researchers working on giant water bugs. There were doubts about its taxonomic status and its range of distribution. The females of this species had not been described. Here, we examined eight females with body length of 30.1-33.6 mm and greatest width of body of 14.1-16.0 $\mathrm{mm}$. The color is dark brown, and the body is flattened. The distribution is apparently related to the Negro river and its tributaries in Amazonas state. This species appears to be relatively abundant in these places. As reported by Roback \& Nieser (1974), the general appearance, structural characters and structure of genitalia place this species in the $B$. bergi group of Lauck (1964). On the other hand, segment 1 of the rostrum is distinctly shorter than 2 and is likely to place it in B. discretum group. Finally, the abdominal sternites completely covered with hair makes $B$. fittkaui very different from other Brazilian species. This species is close to B. hirsutum Roback \& Nieser, 1974 (a Colombian species occurring in Ilanos and Amazon regions), which has the abdomen totally covered with hair and, similar to B. fittkaui, which has segment 1 of he rostrum distinctly shorter than 2 . This is the first record of $B$. fittkaui to Roraima state (Brazil).

\section{Horvathinia pelocoroides Montandon, 1911 (Figs. 3J, 10B)}

Known distribution: ARGENTINA: Misiones, Chaco, Corrientes (Schnack \& Estévez, 2005). BOLIVIA: Chapare, Chipiriri (Schnack \& Estévez, 2005). BRAZIL: São Paulo, Rio Grande do Sul. (Montandon, 1911; Schnack \& Estévez, 2005; Ribeiro et al., 2018). PARAGUAY: Cuá Guazú (Schnack \& Estévez, 2005).

Material examined: 4 males and 1 female, pinned, BRAZIL, Rondônia, near from Pimenta Bueno municipality, Guaporé river, $12^{\circ} 13^{\prime} 19^{\prime \prime} \mathrm{S} 60^{\circ} 32^{\prime} 44^{\prime \prime} \mathrm{W}, 23 . \mathrm{IV} .2006$, J.A. Rafael \& F.F. Xavier Fo colls., T.M. Almeida det.

Notes: This is the first record of Horvathinia to Rondônia state (Brazil).

\section{Lethocerus annulipes (Herrich-Schaeffer, 1845) (Figs. 3F, 11A)}

Known distribution: AMÉRICA DO SUL (HerrichSchaeffer, 1845). ARGENTINA: Buenos Aires, Catamarca, Chaco, Córdoba, Formosa, Misiones (Perez-Goodwyn, 2006). BRAZIL: Amazonas, Bahia, Goiás, Mato Grosso, Minas Gerais, Rio de Janeiro, Rio Grande do Sul, São Paulo, Santa Catarina (Perez-Goodwyn, 2006, 
Ribeiro et al., 2018). GUYANA: Demerara (Nieser, 1975). PARAGUAY: Asunción (Perez-Goodwyn, 2006). SURINAM: Paramaribo, Para (Nieser, 1975). TRINIDAD AND TOBAGO: Puerto Rico and La Española Island (Perez-Goodwyn, 2006). VENEZUELA: unknow State (Perez-Goodwyn, 2006).

Material examined: 1 male, pinned, BRAZIL, Amazonas, Manaus municipality, BR 174, km 50, ZF-2 Reserve, km 21, $02^{\circ} 38^{\prime} 16^{\prime \prime} \mathrm{S} 60^{\circ} 09^{\prime} 26^{\prime \prime} \mathrm{W}, 13-27 . X I I .2012$, F.F. Xavier Fo \& G.Z. Lopes colls., T.M. Almeida, 2018 det.; 1 female, pinned, Manaus municipality, ZF-2 Reserve, km 14, 02³5'21"S $60^{\circ} 06^{\prime} 55^{\prime \prime} \mathrm{W}$, T.M. Almeida, 2013 det. 1 male, pinned, Maranhão, Caxias municipality, $04^{\circ} 39^{\prime} 32^{\prime \prime} \mathrm{S} 43^{\circ} 33^{\prime} 51^{\prime \prime} \mathrm{W}$, in

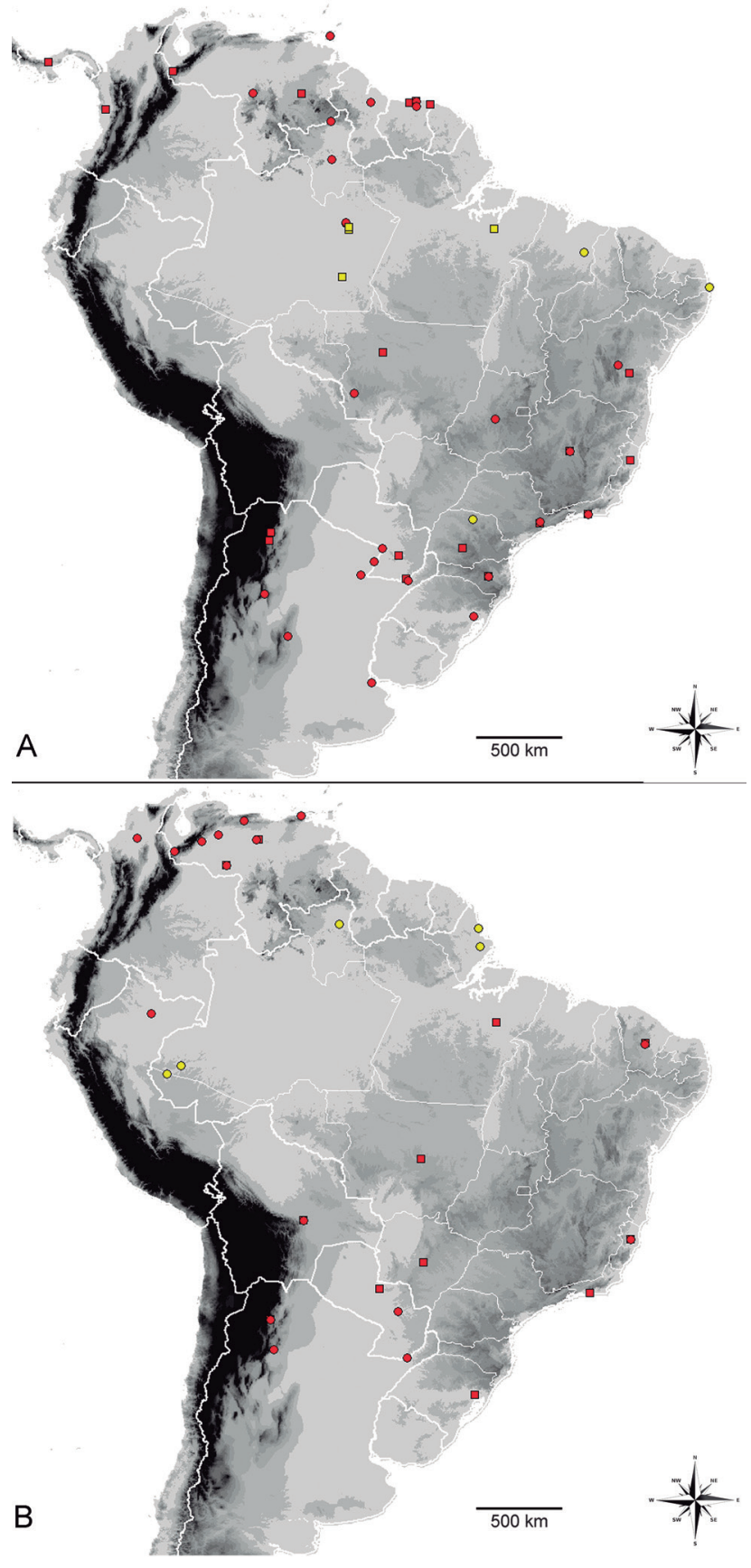

Figure 11. Distribution of Belostomatidae in South America. Red symbol: known records, yellow symbol: new records. $(A)=$ Lethocerus delpontei (square), L. annulipes (circle). (B) = L. paraenses (square), L. maximus (circle). light, 19.V.2007, J.A. Rafael, F.F. Xavier Fo \& F.L. Oliveira colls., T.M. Almeida, 2013 det. 1 male, pinned, Mato Grosso, Nova Lacerda municipality, $14^{\circ} 28^{\prime} 38^{\prime \prime} \mathrm{S} 59^{\circ} 33^{\prime} 30^{\prime \prime} \mathrm{W}$, 27.IV.2006, J.A. Rafael \& F.F. Xavier Fo colls., T.M. Almeida, 2013 det. 1 male, pinned, Paraíba, João Pessoa municipality, 09.X.1976, V. Py-Daniel coll., T.M. Almeida, 2013 det. 1 male, pinned, Paraná, Cambé municipality, 03.X.2012, T.M. Almeida, 2013 det. 1 male, pinned, Roraima, Caracaraí municipality, 14.V.1977, B. Mascarenhas coll., T.M. Almeida, 2013 det.; 4 males and 1 female, pinned, Pacaraima municipality, 25.VI-05.VII.1988, light trap, J.A. Rafael et al. colls., T.M. Almeida, 2013 det.; 1 male, ethyl alcohol 70\%, Amajari municipality, near Uraricoera river, Maracá Ecological Station, 22.IX.1987, team Nhamenhame colls., M.E. Lanzer-de-Souza, 1987 det.

Notes: This is the first record of $L$. annulipes to Maranhão, Paraíba and Paraná states (Brazil).

\section{Lethocerus delpontei De Carlo, 1930 (Figs. 3G, 11A)}

Known distribution: ARGENTINA: Salta, Jujuy, Misiones (De Carlo, 1930; Perez-Goodwyn, 2006). BRAZIL: Bahia, Mato Grosso, Espírito Santo, Minas Gerais, Rio de Janeiro, São Paulo, Paraná, Santa Catarina (Ribeiro et al., 2018). COLOMBIA: Chocó (Perez-Goodwyn, 2006). PANAMA: (Perez-Goodwyn, 2006). PARAGUAY: Villarica (PerezGoodwyn, 2006). SURINAM: Marowijne Saramaca, Paramaribo (Nieser, 1975). VENEZUELA: Bolívar, Tachira (Perez-Goodwyn, 2006).

Material examined: 1 male, ethyl alcohol 70\%, BRAZIL, Amazonas, Caxipacoré river, light trap, 20.IV.1985, E. Bindá coll., 1696, T.M. Almeida, 2013 det.; 2 males, pinned, Manaus municipality, Centro de Projetos e Estudos Ambientais do Amazonas - Cepeam/Soka Gakkai, 0306'44.1"S 59 54'22.7"W, mixed light trap, 04-05.VI.1999, F. Gouveia coll., T.M. Almeida, 2013 det.; 1 male, pinned, Manaus municipality, Aruazinho stream, 06²4'44"S 60²1'25"W, IV.2005, F. Godoi \& A. Lourido colls., T.M. Almeida, 2013 det.; 1 male, ethyl alcohol 70\%, Manaus municipality, Ducke Reserve, Bolívia stream, V. Linard coll., T.M. Almeida, 2017 det. 1 male, ethyl alcohol $70 \%$, Pará, BR 422, Tocantins river, Água Corrente stream, 22.IV.1988, U. Barbosa coll., 1682, T.M. Almeida, 2013 det.

Notes: This is the first record of $L$. delpontei to Amazonas and Pará states (Brazil).

\section{Lethocerus maximus De Carlo, 1938} (Figs. 3H, 11B)

Known distribution: ARGENTINA: Misiones, Salta, Tucumán (De Carlo, 1938; Perez-Goodwyn, 2006). BOLIVIA: Santa Cruz de la Sierra, (Perez-Goodwyn, 2006). BRAZIL: Ceará, Espírito Santo (Perez-Goodwyn, 2006). COLOMBIA: Sucre (Perez-Goodwyn, 2006). PARAGUAY: 
San Pedro (Perez-Goodwyn, 2006). PERU: Huánaco, Loreto (Perez-Goodwyn, 2006). VENEZUELA: Apure, Aragua, Barinas, Guárico, Portuguesa, Sucre, Táchira (Perez-Goodwyn, 2006).

Material examined: 1 male, pinned, BRAZIL, Acre, Cruzeiro do Sul municipality, 07 $36^{\prime} 26^{\prime \prime} S 72^{\circ} 40^{\prime} 55^{\prime \prime} \mathrm{W}$ 20-21.XI.1996, Motta, C.S. et al. colls., T.M. Almeida, 2018 det. 2 males, ethyl alcohol 70\%, Amapá, Tartarugalzinho municipality, N. Hamada \& J.M.C. Calvacante colls., T.M. Almeida, 2013 det.; 1 male, pinned, 02 $31^{\prime} 07.2^{\prime \prime} \mathrm{N}$ $50^{\circ} 58^{\prime} 16.8^{\prime \prime} \mathrm{W}$, light trap, 17.XI.2014, F.F. Xavier Fo coll., T.M. Almeida, 2018 det. 2 males, pinned, Amazonas, Ipixuna municipality, Grande lake, near Gregório river, 0703'11.7"S 7141'40.6"W, 15.V.2011, R. Andreazze \& R.F. Silva colls., T.M. Almeida, 2018 det. 2 males, pinned, Roraima, Boa Vista municipality, 06.IV.1976, E. Faustino coll., T.M. Almeida, 2013 det.

Notes: This is the first record of L. maximus to Acre, Amapá, Amazonas and Roraima states (Brazil).

\section{Lethocerus paraensis Lanzer-de-Souza, 1991 junior synonym of $L$. maximus (Figs. 3I, 11B)}

Known distribution: ARGENTINA: Salta (PerezGoodwyn, 1997). BOLIVIA: Santa Cruz de la Sierra (Perez-Goodwyn, 1997). BRAZIL: Ceará, Espírito Santo, Mato Grosso, Mato Grosso do Sul, Pará, Rio de Janeiro, Rio Grande do Sul (Perez-Goodwyn, 1997). PARAGUAY: Puerto Pinasco (Perez-Goodwyn, 1997). VENEZUELA: Apure, Guárico (Perez-Goodwyn, 1997).

Type Material examined: 1 female holotype, pinned, BRAZIL, Pará, Tucuruí lake, near Tocantins river, Germoplasma island, X.1987, Almir coll., Lanzer-deSouza, 1991 det.

\section{Weberiella rhomboides (Menke, 1965)} (Figs. 3K, 10B)

Known distribution: BRAZIL: Amazonas, Rondônia, Roraima, Mato Grosso (Estévez \& Ribeiro, 2011; Ribeiro et al., 2018). FRENCH GUYANA: unknow Commune (Estévez \& Ribeiro, 2011).

Material examined: 1 male, ethyl alcohol 70\%, BRAZIL, Rondônia, Porto Velho municipality, 08 $43^{\prime} 59.4^{\prime \prime} \mathrm{S}$ $63^{\circ} 55^{\prime} 07.6^{\prime \prime} \mathrm{W}$, Tambaqui stream, near Abunã river, 03.VI.2004, N. Hamada, R.F. Keppler \& J.O. Silva colls., T.M. Almeida, 2013 det.

\section{ACKNOWLEDGMENTS}

We thank the Invertebrate Collection of INPA (Dr. Marcio Luiz de Oliveira, curator) for loan of the specimens, Conselho Nacional de Desenvolvimento Científico e Tecnológico (CNPq) and Fundação de Amparo à Pesquisa do Estado do Amazonas (FAPEAM) - Programa de Apoio a Núcleos de Excelência (Pronex). and CAPES-Pro-equipamentos. We are grateful to Dr. Ana Lía Estevez for literature provided and for training TMA on Belostomatidae taxonomy in Museo de La Plata, Argentina and to Philip M. Fearnside for revising the manuscript. Part of this work was done under INPA's PIBIC program by TMA; TMA is supported by FAPEAM, FS by CAPES Ph.D. fellowship and NH is a CNPq research fellow (Process n. 307849/2014-7).

\section{REFERENCES}

Amyot, C.J.B. \& Serville, J.G.A. 1843. Histoire Naturelle des Insectes Hémiptères. Paris, Librairie Encyclopedique de Roret. Ixxxvi + 681p. 12 pls.

Berg, C. 1879. Hemiptera Argentina Enumeravit Speciesque Novas. Bonariare, Ex Typographiae Pauli E.Coni. 316p.

Chernoff, B.; Willink, P.W. \& Montambault, J.R. 2001. A biological assessment of the aquatic ecosystems of the Río Paraguay basin, Alto Río Paraguay, Paraguay. Washington, Conservation International. 156. (Rap Bulletin of Biological Assessment, 19).

Contartese, A.M. \& Bachmann, A.0. 1987. Distribución geografica de las Belostomatidae argentinas (Heteroptera). Physis (Buenos Aires), 45(108): 25-27.

De Carlo, J.A. 1930. Familia Belostomidae, géneros y especies para la Argentina. Revista de la Sociedad Entomológica Argentina, 3: 110-116.

De Carlo, J.A. 1932. Nuevas especies de belostómidos (Hemiptera). Revista de la Sociedad Entomológica Argentina, 5(22): 121-126.

De Carlo, J.A. 1933. Familia Belostomidae (Hemiptera); descripción de especies nuevas procedentes del Brasil. Boletim do Museu Nacional de Rio de Janeiro, 9(1): 93-98.

De Carlo, J.A. 1938. Los Belostómidos americanos. Anales del Museo Argentino de Ciencias Naturales 'Bernardino Rivadavia' Buenos Aires, 39(155): 189-260, 7 pls.

De Carlo, J.A. 1956. Especies nuevas de la familia Belostomatidae (Hemiptera) y algunas consideraciones sobre otras poco conocidas. Revista de la Sociedad Entomológica Argentina, 19(3-4): 51-56.

De Carlo, J.A. 1958. Identificación de las especies del género Horvathinia Montandon. Descripción de tres especies nuevas (HemipteraBelostomatidae). Revista de la Sociedad Entomológica Argentina, 20(3-4): 45-52.

De Carlo, J.A. 1960. Especies nuevas del género Belostoma y consideraciones sobre otras poco conocidas (Hemiptera-Belostomatidae). Revista de la Sociedad Entomológica Argentina, 22: 47-59.

De Carlo, J.A. 1966. Un nuevo género, nuevas especies y referencias de otras poco conocidas de la familia Belostomidae (Hemiptera). Revista de la Sociedad Entomológica Argentina, 28: 97-109.

Dias-Silva, K.; Cabette, H.S.R.; Juen, L. \& De Marco Jr., P. 2010. The influence of habitat integrity and physical-chemical water variables on the structure of aquatic and semi-aquatic Heteroptera. Zoologia, 27(6): 918-930.

Digiani, M. 2002. Belostomatidae (Insecta: Heteroptera) as intermediate hosts of digenetic trematodes, Comparative Parasitology, 69(1): 89-92.

Dufour, L. 1863. Essai monographique sur les Belostomides. Annales de la Société Entomologique de France, 4(3): 375-400.

Estévez, A.L. 1996. Revision Sistematica del Género Belostoma Latreille (Hemiptera-Heteroptera). Grupos de Belostoma (Belostoma) triangulum, B. (B.) minor, B. (B.) oxyurum, B. (B.) plebejum y B. (B.) denticolle. La Plata, 
Doctor thesis. Facultad de Ciencias Naturales y Museo, Universidad Nacional de La Plata. vi + 117p., 16 pls.

Estévez, A.L. \& Polhemus, J.T. 2001. The small species Belostoma (Hemiptera: Belostomatidae). I. Introduction, key to species groups and a revision of the denticolle group. Iheringia, Série Zoologia, 91: 151-158.

Estévez, A.L. \& Polhemus, J.T. 2007. The small species of Belostoma (Heteroptera, Belostomatidae): revision of the plebejum group. Revista de Biología Tropical, 55: 147-155.

Estévez, A.L. \& Ribeiro, J.I. 2011. Weberiella De Carlo, 1966 (Insecta: Heteroptera: Belostomatidae) revisited: Redescription with a key to the genera of Belostomatidae and considerations on back-brooding behaviour. Zoologischer Anzeiger, 250(1): 46-54.

Heckman, C.W. 1998. Encyclopedia of South American aquatic insects: Hemiptera-Heteroptera. Illustrated keys to known families, genera, and species in South America. New York, Springer Science+Business Media B.V. ix $+679 p$

Herrich-Schaeffer, G.A.W. 1845. Die wanzenartigen Insecten: getreu nach der Natur abgebildet und beschrieben. Nürnberg. Acten Band, Erstes Heft. https://www.biodiversitylibrary.org/item/44188\#page/303/mode/1up.

Lanzer, M.E.B. 1976. 0 gênero Belostoma Latreille, 1807 (HeteropteraBelostomatidae): novas ocorrências para o Estado do Rio Grande do Sul, Brasil. Iheringia, Série Zoologia, 49: 3-6.

Lanzer-de-Souza, M.E. 1991. Nova espécie do gênero Lethocerus Mayr, 1853 (Heteroptera, Belostomatidae, Lethocerinae). Iheringia, Série Zoologia,71: 139-143.

Lanzer-de-Souza, M.E. 1992. Nova espécie do gênero Belostoma Latreille, 1807 (Heteroptera, Belostomatidae). Iheringia, Série Zoologia, 72: 147-150.

Latreille, P.A. 1807. Genera Crustaceorum et Insectorum Secundum Ordinem Naturalem in Familias Disposita, Iconibus Exemplisque Plurimis Explicata. Parisiis et Argentorati, Amand Koenig. v. 3, 259p.

Lauck, D.R. 1962. A monograph of the genus Belostoma (Hemiptera) Part I. Introduction to $B$. dentatum and subspinosum groups. Bulletin of the Chicago Academy of Sciences, 11(3): 34-81.

Lauck, D.R. 1963. A monograph of the genus Belostoma (Hemiptera), Part II. B. aurivillianum, stollii, testaceopallidum, dilatatum, and discretum groups. Bulletin of the Chicago Academy of Sciences, 11(4): 82-101.

Lauck, D.R. 1964. A monograph of the genus Belostoma (Hemiptera) Part III. B. triangulum, bergi, minor, bifoveolatum, and flumineum groups. Bulletin of the Chicago Academy of Sciences, 11(5): 102-154.

Magalhães, C.; Kury, A.B.; Bonaldo, A.B.; Hajdu, E. \& Simone, LR. 2005. Coleções de invertebrados do Brasil. Documento de trabalho. Projeto Diretrizes e Estratégias para a Modernização de Coleções Biológicas Brasileiras e a Consolidação de Sistemas Integrados de Informações sobre Biodiversidade. Available at: http://www.cria.org.br/cgee/documentos/Colecoesdelnver tebradosMagalhaesBonaldoKuryHadju.pdf. Access in 12/06/2018.

Mayr, G. 1863. Hemipterogische Studien. Die Belostomiden. Verhandlungen der Zoologisch-Botanischen Gesellschaft in Wien, 13: 339-364.

Mayr, G. 1871. Die Belostomiden. Verhandlungen der Zoologisch-Botanischen Gesellschaft in Wien, 21: 399-440.

Mayr, G.L. 1853. Zwei neue Wanzen aus Kordofan, Limnogeton fieberi u. Lethrocerus cordofanus. Verhandlungen der Zoologisch-Botanischen Gesellschaft in Wien, 2: 14-18.

Menke, A.S. 1965. A new South American toe biter (Hemiptera, Belostomatidae). Contributions in Science, Natural History Museum of Los Angeles County, 89: 1-3.

Menke, A.S. \& Lauck, D.R. 1962. The Machris Brazilian Expedition-entomology: Belostomatidae (Hemiptera). Contributions in Science, Natural History Museum of Los Angeles County, 55: 3-8.

Montandon, A.L. 1895. Hémiptères Hétéroptères. Première liste et descriptions $d^{\prime}$ espèces nouvelles. Viaggio del Dott. A. Borelli nella
Republica Argentina en el Paraguay. Bolletino dei Musei di Zoologia ed Anatomia comparata della R. Università di Torino, 10: 1-10.

Montandon, A.L. 1899. Hemiptera cryptocerata. s. fam. Mononychinae, notes et descriptions d'espèces nouvelles. lère partie. Bulletin de la Socité des Sciences de Bucarest, Vol. 8, p. 392-407.

Montandon, A.L. 1900. Notes sur quelques Hémiptères Hétéroptères et descriptions d'espèces nouvelles des collections du Musée Civique de Gênes. Annali del Museo Civico di Storia Naturale Giacomo Doria di Genova, 20(40): 1-11.

Montandon, A.L. 1903a. Especes nouvelles ou peu connues du genre Belostoma. Appartennant aux collections du Musée National Hongrois. Annales Musei Nationalis Hungarici, 1: 359-363.

Montandon, A.L. 1903b. Deux nouvelles espèces du genre Belostoma (= Zaitha Amyot et Serville et auct.) des collections du Muséum de Paris. Bulletin du Muséum d'Histoire Naturelle, 9: 21-23.

Montandon, A.L. 1908. Nouvelles espèces d'Hémiptères aquatiques. Annales Historico-Naturales Musei Nationalis Hungarici, 6: 299-304.

Montandon, A.L. 1911. Deux genres nouveaux d'Hydrocorises. Annals of the Museum Nationalis Hungarici, 9: 244-250.

Moreira, F.F.F.; Barbosa, J.F.; Ribeiro, J.R.I. \& Alecrim, V.P. 2011. Checklist and distribution of semi-aquatic and aquatic Heteroptera (Gerromorpha and Nepomorpha) occurring in Brazil. Zootaxa, 2958: 1-74.

Morrone, J.J.; Mazzucconi, S.A. \& Bachmann, A.0. 2004. Distributional patterns of Chacoan water bugs (Heteroptera: Belostomatidae, Corixidae, Micronectidae and Gerridae). Hydrobiologia, 523: 159-173.

Nieser, N. 1975. The water bugs (Heteroptera: Nepomorpha) of the Guyana region. Studies on the Fauna of Suriname and Other Guyanas, 16(81): 88-128.

Nieser, N. \& Melo, A.L. 1997. Os Heterópteros Aquáticos de Minas Gerais. Guia Introdutório com Chave de Identificação para as Espécies de Nepomorpha e Gerromorpha. Belo Horizonte, Ed. UFMG. 177p.

Perez-Goodwyn, P.J. 1997. Lethocerus paraensis, new synonym of L. maximus (Heteroptera: Belostomatidae). Revista de la Sociedad Entomológica Argentina, 56(1-4): 122.

Perez-Goodwyn, P.J. 2006. Taxonomic revision of the subfamily Lethocerinae Lauck \& Menke (Heteroptera: Belostomatidae). Stuttgarter Beiträge zur Naturkund Serie A, 695: 1-71.

Polhemus, J.T. 1995. Nomenclatural and synonymical notes on the genera Diplonychus Laporte and Appasus Amyot \& Serville (Heteroptera: Belostomatidae). Proceedings of the Entomological Society of Washington, 97: 649-653.

Ribeiro J.R.I.; Rodrigues H.D.D. \& Barbosa J.F. 2018. Catálogo Taxonômico da Fauna do Brasil. PNUD. Available at: http://fauna.jbri.gov.br/fauna/ faunadobrasil/2. Access in 26/03/2018.

Ribeiro, J.R.I. 2000. Description of the male of Belostoma foveolatum and new records of $B$. costalimai and $B$. stollii (Heteroptera: Belostomatidae). Entomological News, 111(3): 159-170.

Ribeiro, J.R.I. 2004. Redescription and Taxonomic Considerations of Belostoma gestroi Montandon, 1900 (Heteroptera: Belostomatidae). Studies on Neotropical Fauna and Environment, 39(1): 53-56.

Ribeiro, J.R.I. 2005. Família Belostomatidae Leach, 1815 (Insecta: Hemiptera: Heteroptera): chave e catálogo de identificação para as espécies ocorrentes no Estado do Rio de Janeiro, Brasil. Arquivos do Museu Nacional, Rio de Janeiro, 63: 247-262.

Ribeiro, J.R.I. 2007. A review of the species of Belostoma Latreille, 1807 (Insecta, Heteroptera, Belostomatidae) from the four southeastern Brazilian states. Zootaxa, 1477: 1-70.

Ribeiro, J.R.I. \& Alecrim, V.P. 2008. Duas novas espécies de Belostoma Latreille, 1807 (Hemiptera, Heteroptera: Belostomatidae) do grupo plebejum sensu Nieser, 1975. Acta Amazonica, 38: 179-188. 
Ribeiro, J.R.I. \& Estévez, A.L. 2009. The small species of Belostoma Latreille (Heteroptera, Belostomatidae). III. A revision of the oxyurum group, with a new species from Brazil and description of the male of $B$. noualhieri Montandon. Revista Brasileira de Entomologia, 53: 207-215.

Ribeiro, J.R.I.; Estévez, A.L.; Moreira, F.F.F. \& Guilbert, E. 2017. Revision of the Belostoma dentatum group sensu Nieser (Insecta, Heteroptera, Belostomatidae). Zootaxa, 4276(2): 177-203.

Roback, S.S. \& Nieser, N. 1974. Aquatic Hemiptera (Heteroptera) from the Llanos of Colombia. Proceedings of the Academy of Natural Sciences of Philadelphia, 126(4): 38-40.

Ruffinelli, A. \& Pirán, A.A. 1959. Hemipteros heterópteros del Uruguai. Boletin de la Facultad de Agronomia de Montevideo, 51: 45-47.

Schnack, J.A. 1976. Los Belostomatidae de la Republica Argentina (Hemiptera). In: Ringuelet, R.A. (Ed.). Fauna de Agua Dulce de la Republica Argentina. Buenos Aires, Fecic. v. 35, Fasciculo 1, p. 7-66.
Schnack, J.A. \& Estévez, A.L. 2005. On the taxonomic status of the genus Horvathinia Montandon (Hemiptera: Belostomatidae). Zootaxa, 1016: 21-27.

Schuh, R.T. \& Slater, J.A. 1995. True Bugs of the World (Hemiptera: Heteroptera). New York, Cornell University Press. 337p.

Stål, C. 1860. Bidtrag Till Rio Janeiro-Traktens Hemipter-Fauna. Kungliga Svenska Vetenskapsakademies Handlingar, 2(7): 1-84.

Taddei, W.; Martins, U.R.; Vivo, M. \& Percequillo, A.R. 1999. 0 acervo das coleções zoológicas do estado de São Paulo. In: Joly, C.A. \& Bicudo, C.E.M. (Eds.). Biodiversidade do estado de São Paulo, Brasil: síntese do conhecimento ao final do século XX, 7: Infraestrutura para conservação da biodiversidade. São Paulo, FAPESP. p. 1-109.

Uhler, P.R. 1884. Order VI. Hemiptera. In: Kingsley, J.S. (Eds.). The Standard Natural History. Vol. II. Crustacea and insects. Boston, Mass., E. Cassino \& Co. p. 204-296. 